\title{
Dynamic phasor based frequency scanning for grid-connected power electronic systems
}

\author{
M K DAS* and A M KULKARNI \\ Department of Electrical Engineering, Indian Institute of Technology Bombay, Mumbai 400076, India \\ e-mail: das123mukesh@gmail.com; anil@ee.iitb.ac.in
}

MS received 13 June 2015; revised 1 December 2016; accepted 31 January 2017; published online 11 October 2017

\begin{abstract}
Frequency scanning is a method of obtaining the frequency response of a system by injecting a small-amplitude wide-band signal as an input in a time domain simulation of the system. This is an alternative to analytical derivation of small-signal models, especially for complex grid-connected power electronic systems (PESs). These models are required for the study of adverse interaction of PES with lightly damped oscillatory modes in a power system. The use of the frequency scans for conventional small-signal stability analysis is predicated upon the time-invariance of the underlying model. Since PES are generally time-periodic, timeinvariance may be achieved in some transformed variables. Although the DQ transformation is suitable in many situations, it is not so for systems with low-order harmonics, individual-phase schemes, unbalanced or singlephase systems, and PES with negative-sequence controllers. This paper proposes the use of dynamic phasor variables in such situations since the underlying model in these variables is time-invariant. The procedure for dynamic phasor based scanning is, however, intricate because wide-band signal injection results in the simultaneous presence of harmonic dynamic phasor components. The paper outlines this procedure and presents illustrative case studies of Thyristor Controlled Series Compensator (TCSC) and STATCOM. For the TCSC, a comparison of the frequency response obtained from the scanning method and the one obtained from an approximate analytical dynamic phasor model is also presented.
\end{abstract}

Keywords. Frequency scanning; harmonic stability; impedance-based analysis; dynamic phasors; powerelectronics-based power system.

\section{Introduction}

Power electronic systems (PES) are increasingly being deployed in power systems in the form of HVDC systems, FACTS controllers and grid-interfaces for renewable energy sources. These devices facilitate flexible operation and control of power systems. Power electronic converters generate harmonics and use fast-acting controllers, because of which there is potential for adverse interactions with the lightly damped oscillatory modes of the power system. Adverse interactions can be manifested as harmonic magnification [1], unstable torsional oscillations [2], or network-controller mode instabilities [3].

Therefore, it is important to develop modelling and analytical tools for the study of these adverse interactions in grid-connected PES. One of the ways to analyse such problems is the use of time-domain electro-magnetic transient simulations. Such simulations use detailed models of PES. A large number of simulations are required for the study of aspects like the effect of parameter variations and

*For correspondence the study of different contingencies. This is time-consuming and the inferences may be case specific.

Analysis of the linearized state-space model of the system can give a better insight into the effect of system parameters. Tools like eigen-value analysis and Nyquist criterion are useful for this purpose, but are applicable only for Linear Time Invariant (LTI) systems. Dynamic models for many PES may be developed by neglecting harmonics and applying a time-variant DQ transformation to the phase variables [4]. Alternative formulations based on Dynamic Phasors [5] and Poincare-mapping based discrete time models [6] have also been proposed. The derivation of analytical models is tedious when the converter, controllers and synchronization schemes have to be represented accurately. An additional step of linearization is also required, since most models are non-linear. Generic models may not always be available, as many control schemes of PES are not standardized. Modelling of switching action in case of thyristor-based circuits is complicated by the fact that turn-off instants are determined by the current through the devices. Therefore, rigorous analytical model development in a realistic situation is often impractical. 
Frequency scanning is a pragmatic alternative, which involves the use of a time-domain simulation program to numerically obtain the small-signal frequency response of a system [7]. In this technique, a small-amplitude, wide-band periodic signal is injected as an input in the time-domain simulation of the system. The small-signal frequency response of the system is obtained in the periodic steady state, by computing the frequency components of the input signal and the output variables. Examples of inputs and outputs are current and voltage (to obtain the response of the impedance), and generator speed and electrical torque (to obtain synchronizing/damping torques). With this approach, there are no restrictions on the extent of detail in the system model. A user need not have the complete details of the underlying model - the model is probed/ observed only at available inputs/outputs. Moreover, manufacturers often provide highly accurate black-box simulation models to their clients, and it is impossible to know the details of the controllers. In such situations, a simulation-based frequency scan is the only option.

The frequency response/transfer function concept is well understood for systems. For such systems, further use of the frequency response for stability analysis is also straightforward [8-11]. While small-signal injection ensures the extraction of a linearized model, the time-invariance property depends on the system model and the variables used. Frequency scans obtained using variables in which the model is time-variant are problematic, as they are difficult to interpret and utilize. For such systems, the frequency response at one frequency may be distorted because of the interference due to an injection at another frequency.

Fortunately, most PES are time-periodic systems. Therefore, time-invariance may be achieved in some transformed variables. Although the DQ transformation is suitable when switching harmonics in the frequency range of interest are negligible (e.g., a PWM-switched STATCOM analysed for low-frequency transients), this may not be so for systems with low-order harmonics, systems using individual-phase [12] or sequence-based controllers [13], unbalanced systems [14] and single-phase systems. In such situations, the use of dynamic phasor variables may yield the desired time-invariance. Therefore they are the most general variables for frequency scanning of PES. Dynamic phasor based models are of infinite order (corresponding to an infinite number of harmonic phasors), but a reduced order is usually adequate for most applications.

The main contribution of this paper is to propose the use of dynamic phasor variable based frequency scanning and to present in detail, the methodology to obtain the same using wide-band signal injection. Using the examples of STATCOM and Thyristor Controlled Series Compensator (TCSC), it is shown that for systems with negative-sequence controllers and low order harmonics, this scanning yields a smooth frequency response that is independent of the frequency content of the multi-sine input. This indicates the underlying time-invariance of a dynamic phasor model.
For a TCSC, the frequency response obtained from a frequency scan is compared with the one obtained from an approximate analytical dynamic phasor model [5]. The paper also presents the use of dynamic phasor model of PES obtained by frequency scanning for grid interaction studies.

The paper is organized as follows. In section 2, we describe the existing techniques for frequency scanning using phase, sequence and DQ variables and their limitations. Section 3 describes the concept of Dynamic Phasors. Section 4 presents the methodology for obtaining frequency scans in these variables. Section 5 presents case studies that illustrate the frequency responses of STATCOM and TCSC obtained using this method. Grid interaction studies using dynamic phasor models are described in section 6 .

\section{The frequency scanning technique}

In a time-domain simulation of a PES, a small-amplitude voltage or current may be injected in order to get the frequency response of admittance or impedance, respectively, as shown in figure 1(a) and 1(b). This injection is superimposed upon the existing sources, which are required to set up the equilibrium conditions around which the response is obtained.

The injection of a sinusoidal voltage or current may be done one frequency at a time. Each simulation has to be carried out for a sufficient duration so that the natural transients die out and the steady state is reached. This can be time-consuming. Therefore, instead of obtaining the steady state response for each frequency separately, a multisine injection signal of the following form is generally used:

$$
u(t)=a \sum_{l=l_{o}}^{N} \sin \left(2 \pi f_{d} l t+\delta_{l}\right) .
$$

All the frequency components of $u(t)$ are multiples of $f_{d}$. Therefore, $u(t)$ is periodic with a time period $T_{d}=\frac{1}{f_{d}}$. Since

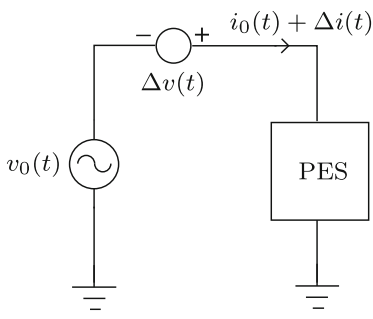

(a)

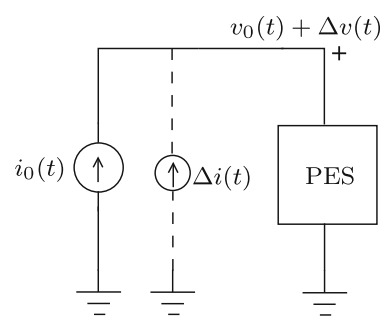

(b)
Figure 1. Voltage- and current injection based frequency scanning. 
$f_{d}$ is the interval between successive frequency components of $u(t)$, it indicates the resolution of the scan.

Since PES along with their controllers are generally nonlinear systems, the amplitude of $u(t)$ must be small so that the small signal frequency response of the linearized system is obtained. Although ' $a$ ' itself could be chosen to be very small, it may introduce errors due to finite precision. Moreover, the remnant natural response of the system, which never truly becomes zero in a finite duration, may also become significant compared to the steady state response due to $u(t)$. This may cause distortions in the frequency scan. Clearly, there is a trade-off among the magnitude of $a$, duration of the scan and the accuracy of the frequency response. For a given $a$, one can reduce the overall amplitude of $u$ by choosing $\delta_{l}$ to have a quadratic dependence on $l$, as in the Schroeder multi-sine [15]:

$$
\delta_{l}=-\frac{\left(l-l_{o}\right)\left(l-l_{o}+1\right)}{\left(N-l_{o}+1\right)} \pi .
$$

After the natural transients have (practically) died down, a window of period $T_{d}$ is stored, and a Fast Fourier Transform (FFT) [16] of both the input and output signals is done in order to obtain the frequency response.

\subsection{Scanning with phase/sequence variables}

For three-phase systems, one may obtain the frequency response by phase-wise injection (one phase at a time). Alternatively, one may obtain the response in the sequence variables, which are defined by the following transformation.

$$
\left[\begin{array}{l}
x_{a} \\
x_{b} \\
x_{c}
\end{array}\right]=\sqrt{\frac{1}{3}}\left[\begin{array}{ccc}
1 & 1 & 1 \\
e^{-j \frac{2 \pi}{3}} & e^{j \frac{2 \pi}{3}} & 1 \\
e^{-j \frac{4 \pi}{3}} & e^{j \frac{4 \pi}{3}} & 1
\end{array}\right]\left[\begin{array}{c}
x_{+} \\
x_{-} \\
x_{o}
\end{array}\right]
$$

The scheme for sequence-based scanning is shown in figure 2. Sequence- or phase-based scanning is suitable for passive time-invariant systems like capacitor/filter banks and transmission lines.

Illustrative example: Sequence impedance model of a distributed parameter transmission line. Consider a $500 \mathrm{kV}$ transmission line that is connected to voltage sources at either end, with a $100 \mathrm{MVAr}$ shunt reactor connected at its midpoint-see figure 3. The transmission line is represented by the frequency-dependent phase model given in PSCAD [17]. The tower configuration 'Tower:3LVert' of PSCAD is used. The frequency responses of the network impedances obtained by frequency scanning using sequence variables are shown in figure 4 . The multi-sine parameters are $a=1 \mathrm{kV}$ and $f_{d}=1 \mathrm{~Hz}$. The frequency scanning range is from $1 \mathrm{~Hz}$ to $350 \mathrm{~Hz}$. The given network has a parallel resonance at $250 \mathrm{~Hz}$. Non-zero coupling terms in the frequency responses are present since the

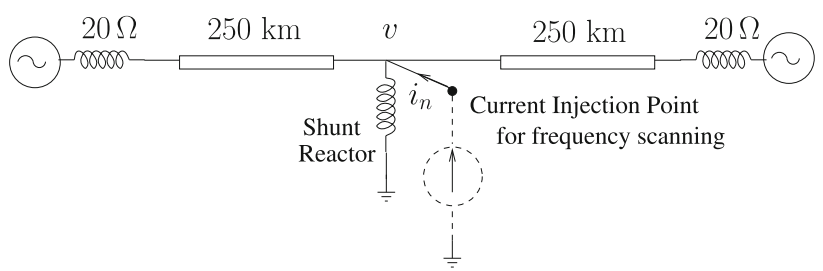

Figure 3. The transmission network under consideration.
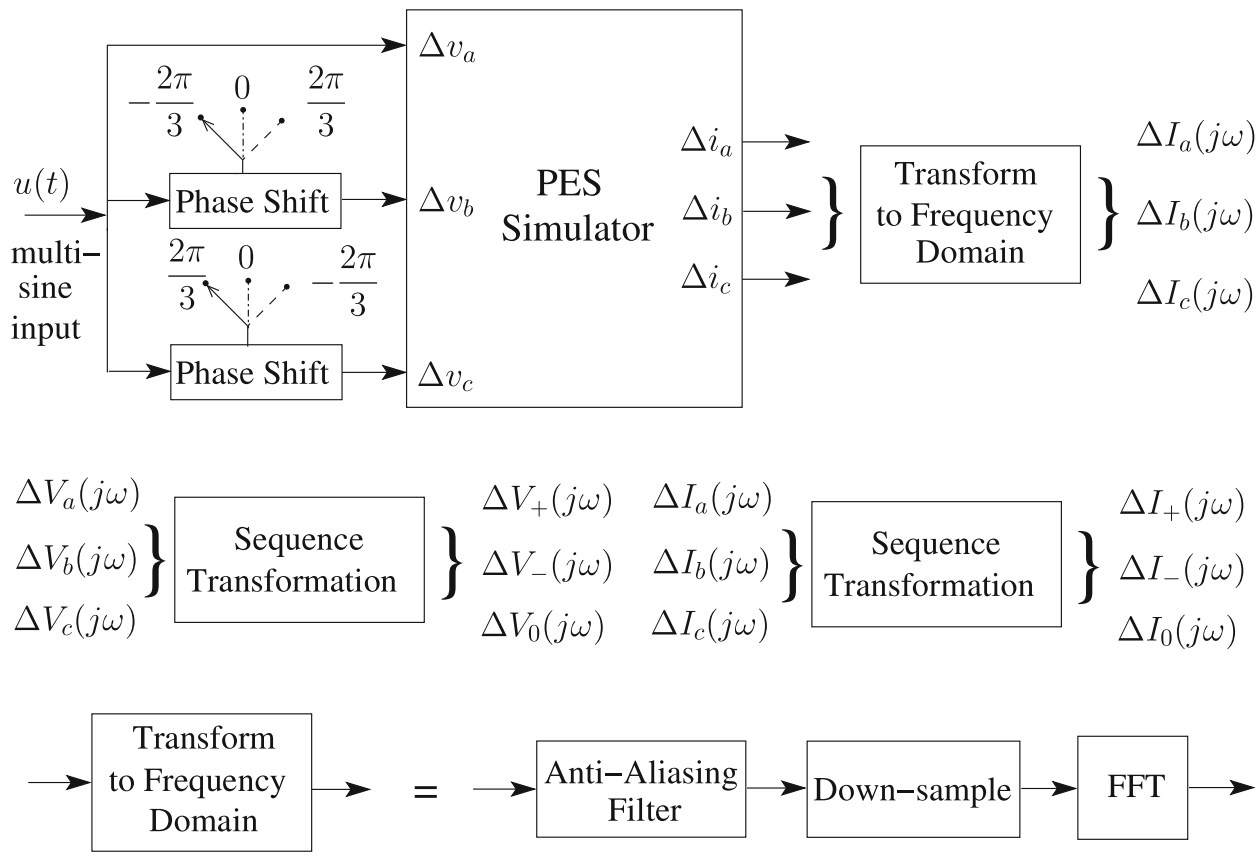

Figure 2. Frequency Scanning based on sequence variables. Note that three scans are required, with different phase shifts. 

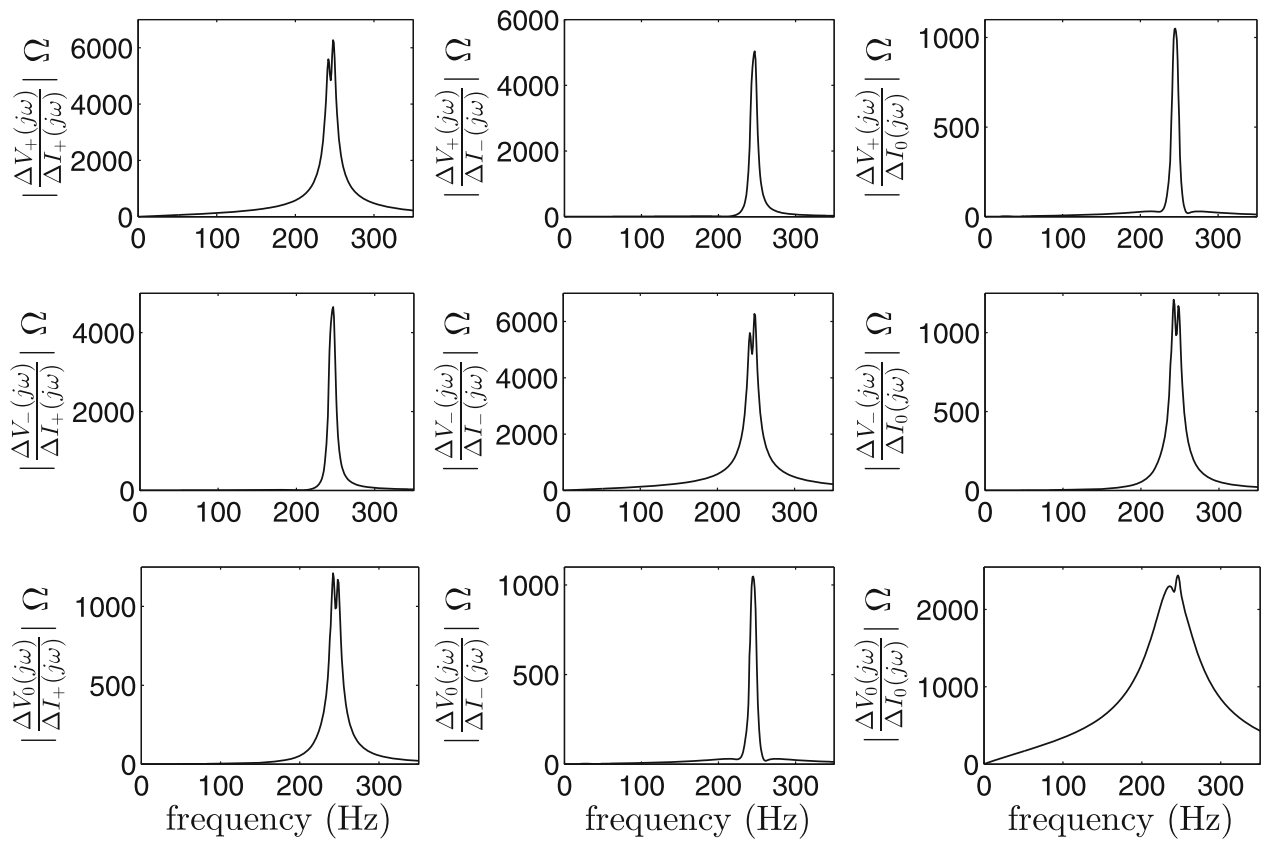

Figure 4. Frequency response of the transmission network.

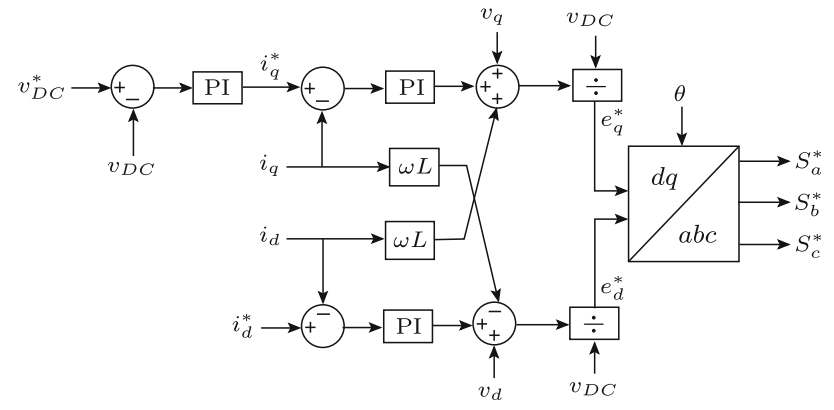

Figure 5. STATCOM type-I controller [4].

conductors are placed in an unsymmetrical fashion on the tower leading to sequence coupling.

\subsection{Limitations of phase/sequence variable based scans}

Phase/sequence-based frequency scanning cannot be directly used for PES that are time-variant when expressed in terms of phase or sequence variables. As an example, consider a \pm 200 MVAr, STATCOM equipped with 'Type-I' controller [4] as shown in figure 5. In the figure, the variables shown with lower case subscripts 'd' and 'q' refer to the local rotating frame, the reference for which is obtained by a PLL tracking the STATCOM bus voltage $v$; ' $\theta$ ' is the output generated by this PLL; $v_{D C}$ is the dc link voltage, whereas $i$ represents the current. $S_{a}^{*}, S_{b}^{*}$ and $S_{c}^{*}$ represent the modulating signals used for PWM pulse generation.
The STATCOM is connected to a $500 \mathrm{kV}, 50 \mathrm{~Hz}$ threephase voltage source through a $500 \mathrm{kV} / 25 \mathrm{kV}$ converter transformer. The STATCOM uses sine-triangle PWM to generate firing pulses, with a carrier frequency of $1650 \mathrm{~Hz}$. For obtaining the frequency scan, a multi-sine voltage signal, $\Delta v(t)$, is injected as shown in figure 6(a), with $a=0.7 \mathrm{kV}$. The frequency scanning range is from 1 to $150 \mathrm{~Hz}$ with $f_{d}=1 \mathrm{~Hz}$. The complete system is simulated using PSCAD-EMTDC [17].

The frequency response of positive sequence admittance is shown in figure 6(b). A distortion is seen in the frequency range 1-100 Hz. This distortion can be explained as follows. The model of a STATCOM in phase or sequence variables is time-variant, involving multiplicative switching functions [18], which have fundamental and harmonic components. The injection of a positive sequence voltage at a frequency $\omega$ will cause negative and positive sequence currents at $\omega-2 \omega_{o}$ and $\omega$, respectively, due to the fundamental component of the switching function. Moreover, if $\omega<2 \omega_{o}$, then one would effectively have two positive sequence components at $\omega$ and $2 \omega_{o}-\omega$ due to sequence flipping [19]. The latter can interfere with an additional positive sequence output, which will be present if there is a multi-sine injection component at $2 \omega_{o}-\omega$.

This is also illustrated in figure 7 for the STATCOM, for a single frequency injection at $20 \mathrm{~Hz}$ in positive sequence voltage. The $20 \mathrm{~Hz}$ signal causes positive sequence current components at 20 and $80 \mathrm{~Hz}$ ( $-80 \mathrm{~Hz}$ in negative sequence) and no negative sequence current in the positive frequency range.

Similarly, the injection of a negative sequence voltage at a frequency $\omega$ will cause positive and negative sequence 


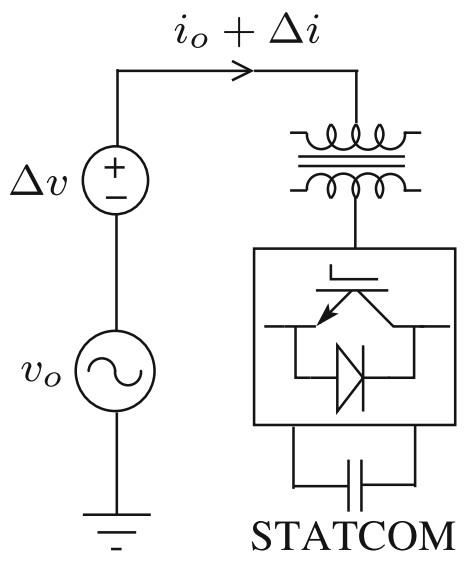

(a)

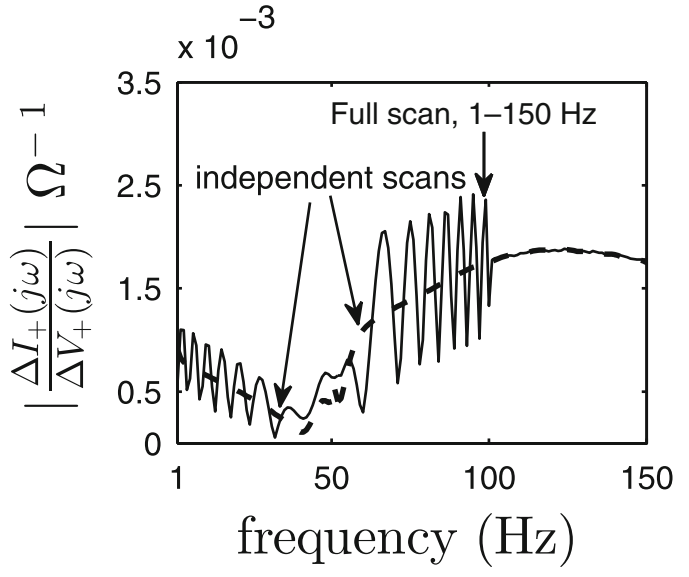

(b)

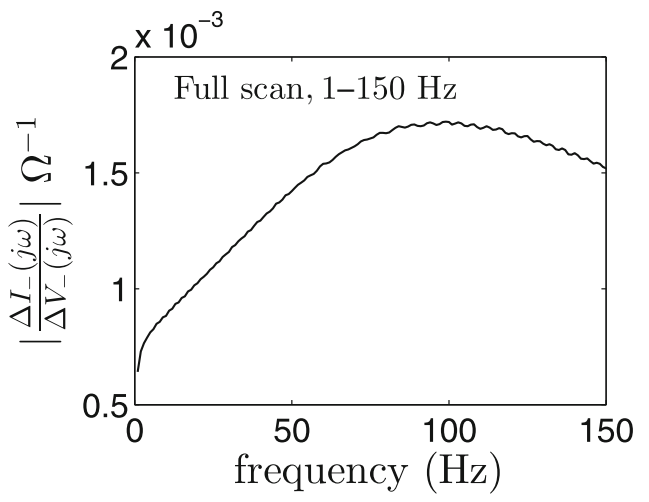

(c)

Figure 6. (a) Frequency scanning scheme for a STATCOM, (b) frequency response of positive sequence admittance and (c) frequency response of negative sequence admittance.

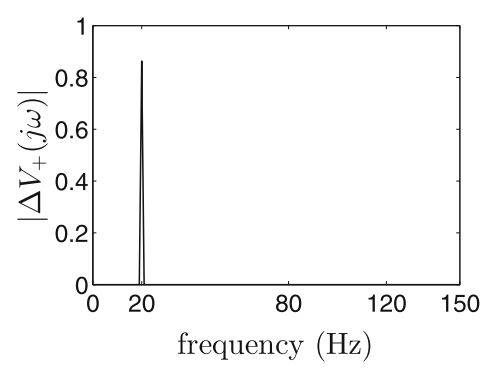

(a)

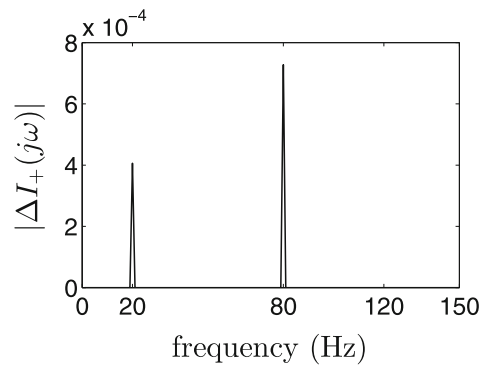

(b)

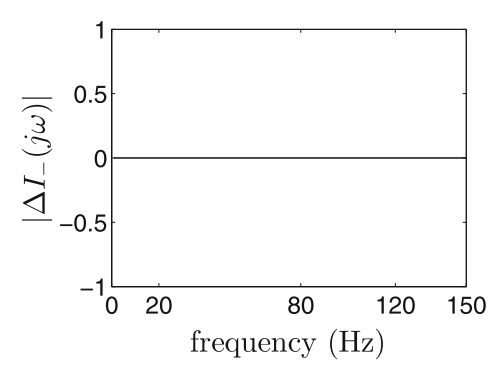

(c)

Figure 7. Frequencies seen in the positive and negative sequence current when a positive sequence sinusoidal signal is injected at a single frequency, $20 \mathrm{~Hz}$.

currents at $\omega+2 \omega_{o}$ and $\omega$, respectively, due to the fundamental component of the switching function. This is illustrated in figure 8 , for a single frequency injection of negative sequence voltage at $20 \mathrm{~Hz}$. In this case, there are no additional frequency components in the positive and negative sequence currents. A negative sequence scan, therefore, does not have 'interference' (see figure 6(c)).
Distortions seen in frequency response of the positive sequence admittance can be circumvented by limiting the frequencies in a single scan (e.g. independent scans are done from 0 to $\omega_{0}, \omega_{0}$ to $2 \omega_{0}$ and so on) as shown in figure 6(b). Although this gets rid of the interference, the theoretical implications of the use of such scans for stability analysis is unclear. 


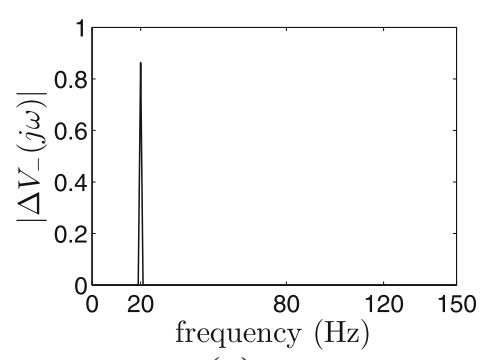

(a)

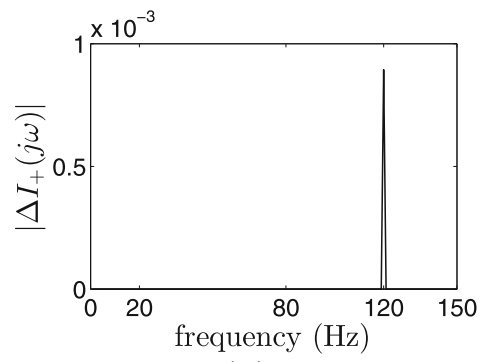

(b)

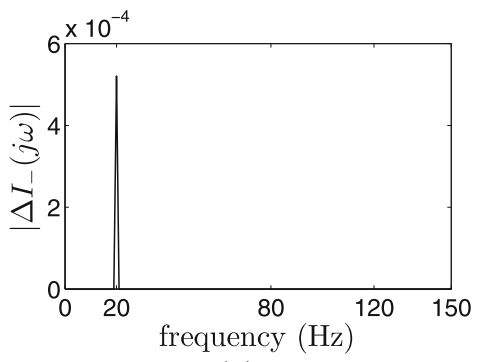

(c)

Figure 8. Frequencies seen in the positive and negative sequence current when a negative sequence sinusoidal signal is injected at a single frequency, $20 \mathrm{~Hz}$.

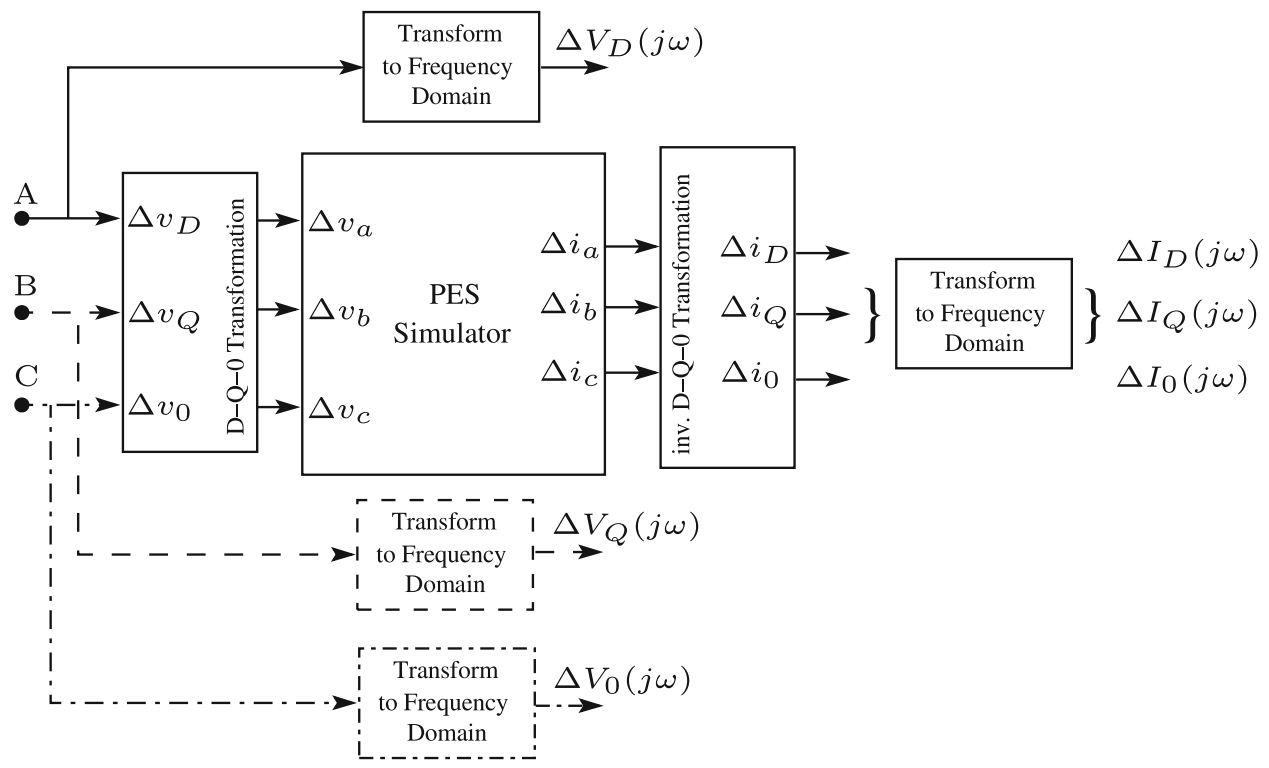

Figure 9. Scheme based on DQ components. Three independent frequency scans are done: (1) by applying the multi-sine signal $u(t)$ at the point $\mathbf{A}$ keeping $\mathbf{B}$ and $\mathbf{C}$ at zero, (2) by applying $u(t)$ at $\mathbf{B}$, keeping $\mathbf{A}$ and $\mathbf{C}$ at zero and (3) by applying $u(t)$ at $\mathbf{C}$, keeping $\mathbf{A}$ and $\mathbf{B}$ at zero.

\subsection{Scanning with $D Q$ variables}

If switching is done in a balanced fashion and switching harmonics are low, then the model of devices like a STATCOM are time-invariant in DQ variables [4]. The DQ transformation is defined as follows:

$$
\left[\begin{array}{l}
x_{a} \\
x_{b} \\
x_{c}
\end{array}\right]=\sqrt{\frac{2}{3}}\left[\begin{array}{ccc}
\cos (\gamma) & \sin (\gamma) & \frac{1}{\sqrt{2}} \\
\cos \left(\gamma-\frac{2 \pi}{3}\right) & \sin \left(\gamma-\frac{2 \pi}{3}\right) & \frac{1}{\sqrt{2}} \\
\cos \left(\gamma-\frac{4 \pi}{3}\right) & \sin \left(\gamma-\frac{4 \pi}{3}\right) & \frac{1}{\sqrt{2}}
\end{array}\right]\left[\begin{array}{c}
x_{D} \\
x_{Q} \\
x_{o}
\end{array}\right]
$$

$\gamma=\omega_{o} t$, where $\omega_{o}$ is the operating frequency. The scheme of DQ based injection [8] is shown in figure 9. Frequency scanning set-up in PSCAD [17] is shown in figure 10 (Note that any EMTP like software can be used for this purpose). From PSCAD simulation, time-domain response of both voltage and current in DQ variables are obtained. FFT operation in MATLAB [20] is used to extract frequency components from these voltage and current responses.

The frequency response of the DQ admittance model of the STATCOM considered earlier is shown in figure 11. There is no interference seen in the frequency scans due to the timeinvariance of the underlying model in the DQ variables. DQbased models are also time-invariant for balanced passive systems and for balanced rotational machines [2].

\subsection{Relationship between $D Q$ model and sequence model}

The DQ admittance model is in the form of a matrix transfer function as shown below: 

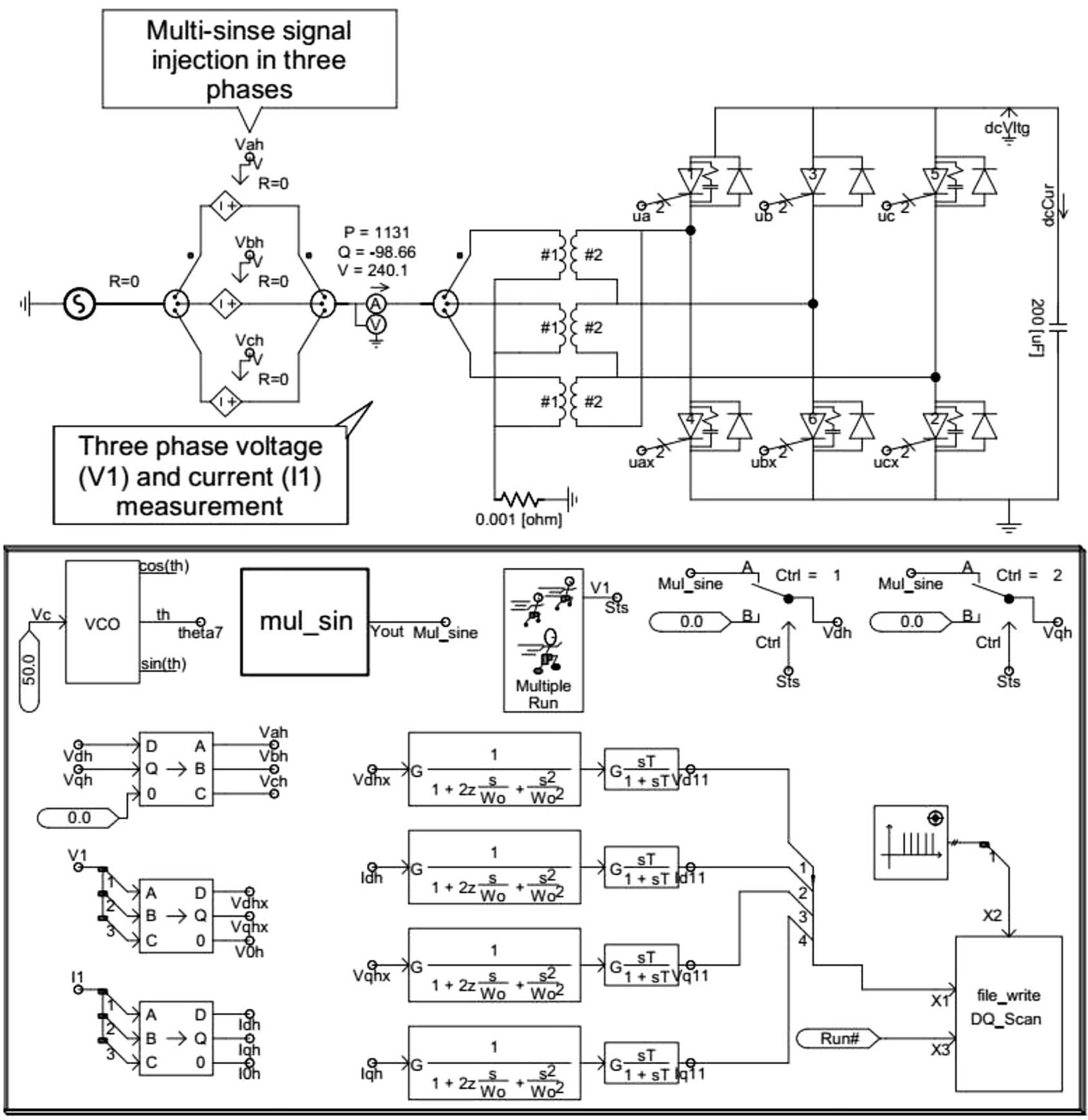

1. Multi-sine signal generation and

2. Selector for injecting in $D$ and $Q$ component separately

3. Reference angle generation for $D Q$ transfromation

4. $A B C$ to $D Q$ conversion of voltage and current

5. Data filtering and down-sampled for data storage

Figure 10. Frequency scanning set-up in PSCAD. 

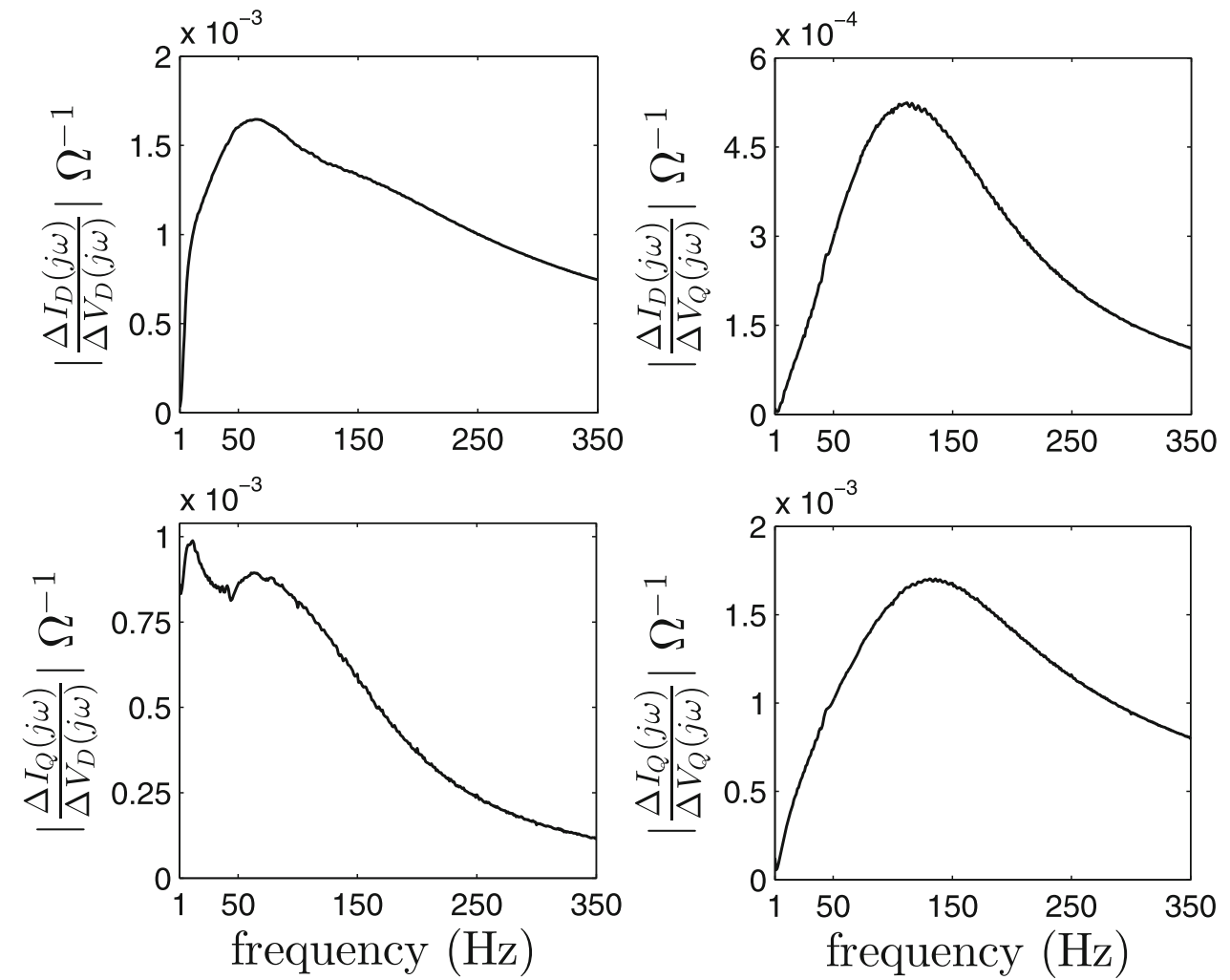

Figure 11. Frequency response of STATCOM obtained from a DQ-based scan.

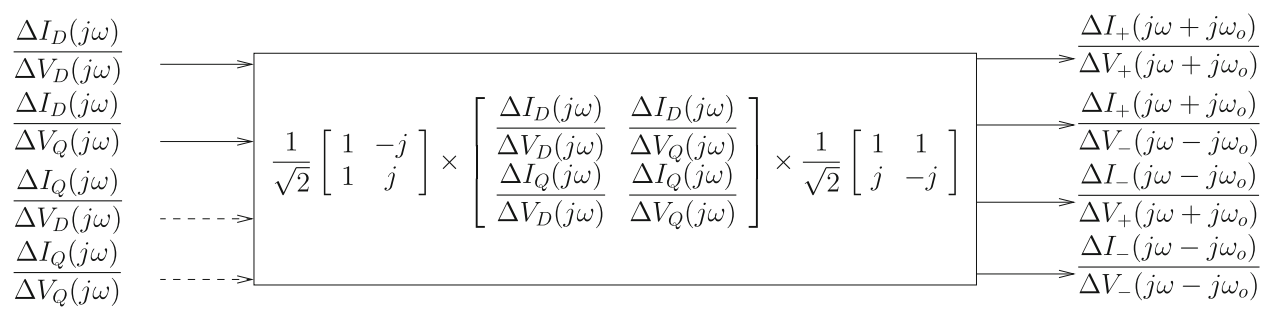

Figure 12. Conversion from DQ model to the corresponding sequence model.

$$
\left[\begin{array}{l}
I_{D}(j \omega) \\
I_{Q}(j \omega)
\end{array}\right]=\underbrace{\left[\begin{array}{ll}
Y_{D D}(j \omega) & Y_{D Q}(j \omega) \\
Y_{Q D}(j \omega) & Y_{Q Q}(j \omega)
\end{array}\right]}_{Y_{D Q}}\left[\begin{array}{l}
V_{D}(j \omega) \\
V_{Q}(j \omega)
\end{array}\right] .
$$

Using (a) $e^{j \theta}=\cos \theta+j \sin \theta$ and (b) the frequency shift property of the frequency response: $X(j \omega) \leftrightarrow x(t)$ implies $X\left(j \omega-j \omega_{o}\right) \leftrightarrow e^{j \omega_{o} t} x(t)$, the DQ variables and sequence variables can be related to each other in the frequency domain by the following equation:

$$
\left[\begin{array}{l}
X_{D}(j \omega) \\
X_{Q}(j \omega)
\end{array}\right]=\frac{1}{\sqrt{2}}\left[\begin{array}{cc}
1 & 1 \\
j & -j
\end{array}\right]\left[\begin{array}{l}
X_{+}\left(j \omega+j \omega_{o}\right) \\
X_{-}\left(j \omega-j \omega_{o}\right)
\end{array}\right] .
$$

Using this, a DQ model can be converted to the corresponding sequence model as shown in figure 12 . Note that there may be a complementary-frequency coupling between the positive and negative sequence variables (this is true for devices like STATCOM [4]). For a STATCOM, a negative sequence injection of voltage at a frequency $\omega-\omega_{o}$ results in currents at frequencies $\omega-\omega_{o}$ and $\omega+\omega_{o}$. This is a manifestation of the fact that the PES state equations are not time-invariant if they are formulated in terms of the sequence variables.

Figure 13 shows the frequency response of the sequence model of STATCOM obtained from its DQ model.

\subsection{Limitations of $D Q$ variable based scans}

If a three-phase system is not balanced, or if harmonics are not negligible, then DQ models may not be time-invariant. 

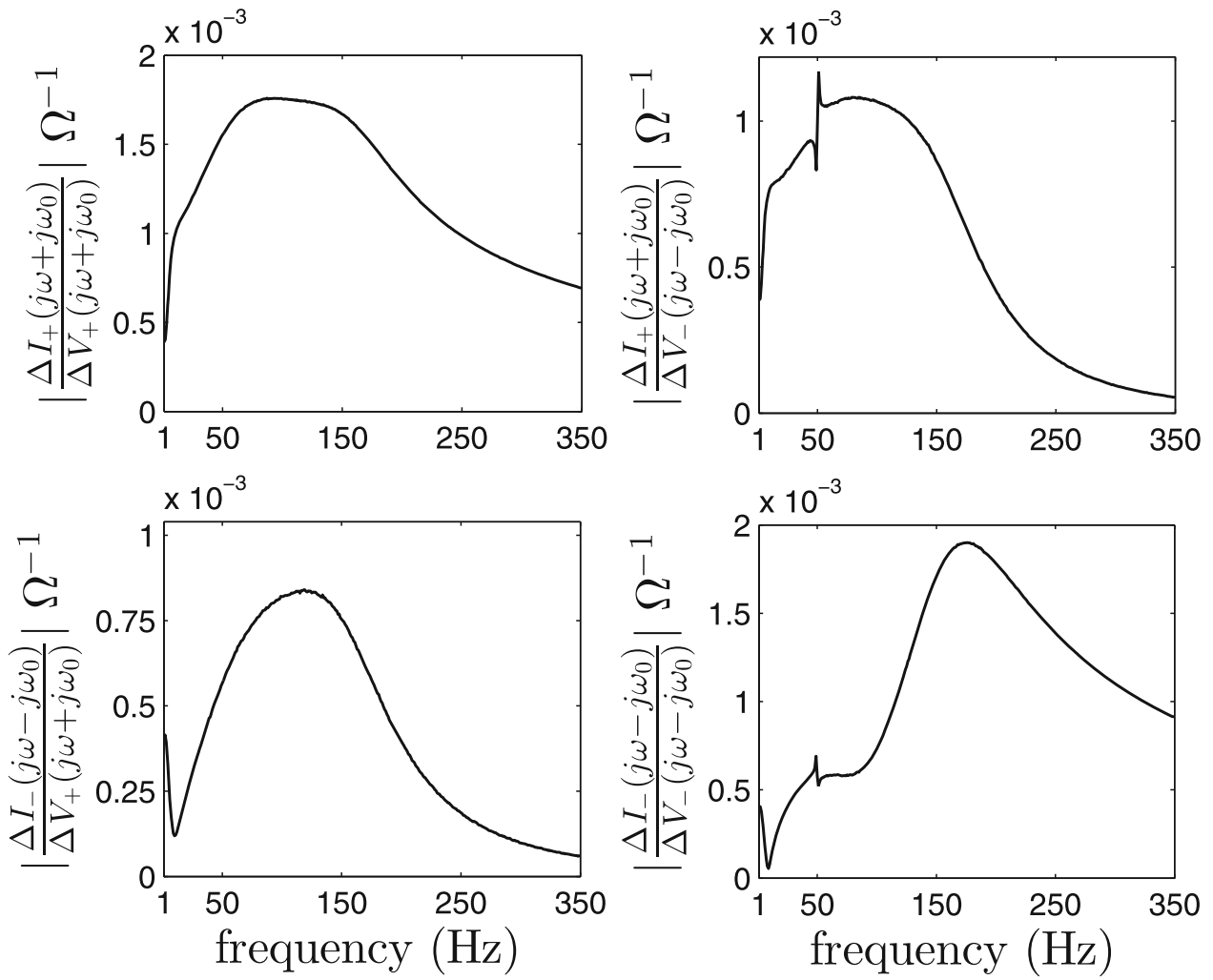

Figure 13. Frequency response of the sequence model of STATCOM obtained from its DQ-based scan.

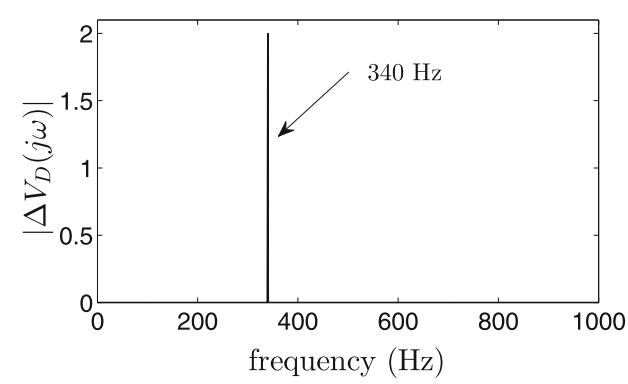

(a)

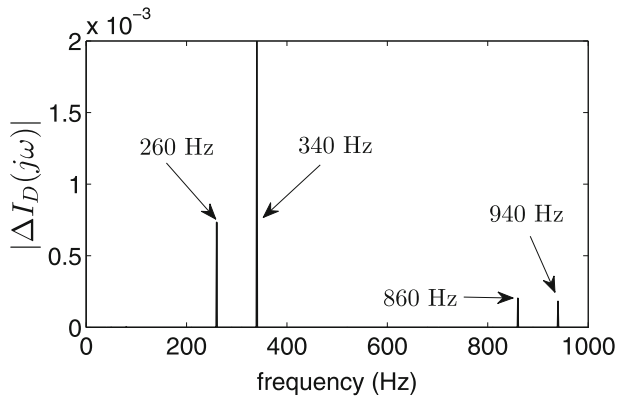

(b)

Figure 14. Twelve-pulse STATCOM: frequencies seen in the D-component of the current when the inject voltage $\Delta v_{D}$ is a sinusoidal signal at a single frequency, $20 \mathrm{~Hz}$.

In such cases, distortions in the frequency response may result. This is illustrated using two examples.

2.5a Twelve-pulse STATCOM: PES like 12-pulse converters and TCSC have significant lower order switching harmonics. For a 12-pulse STATCOM, a significant switching harmonic is present at $600 \mathrm{~Hz}$ (in the DQ frame with fundamental frequency of $50 \mathrm{~Hz}$ ). Thus a sinusoidal injection at a frequency $f$ will cause additional components at $600 \pm f$. This is illustrated in figure 14 , for a single frequency injection of $\mathrm{D}$-channel voltage at
$340 \mathrm{~Hz}$. Consequently, a scan using a multi-sine signal having frequencies from 1 to $350 \mathrm{~Hz}$ will cause additional components at the sum and difference frequencies (i.e. $250-600 \mathrm{~Hz}$ and $600-950 \mathrm{~Hz}$ ). These additional components between 250 and $600 \mathrm{~Hz}$ interact with the frequency components between 250 and $350 \mathrm{~Hz}$ of the wide-band signal and causes interference in the frequency scan.

2.5b STATCOM with negative sequence controller: A conventional STATCOM controller may be modified by 


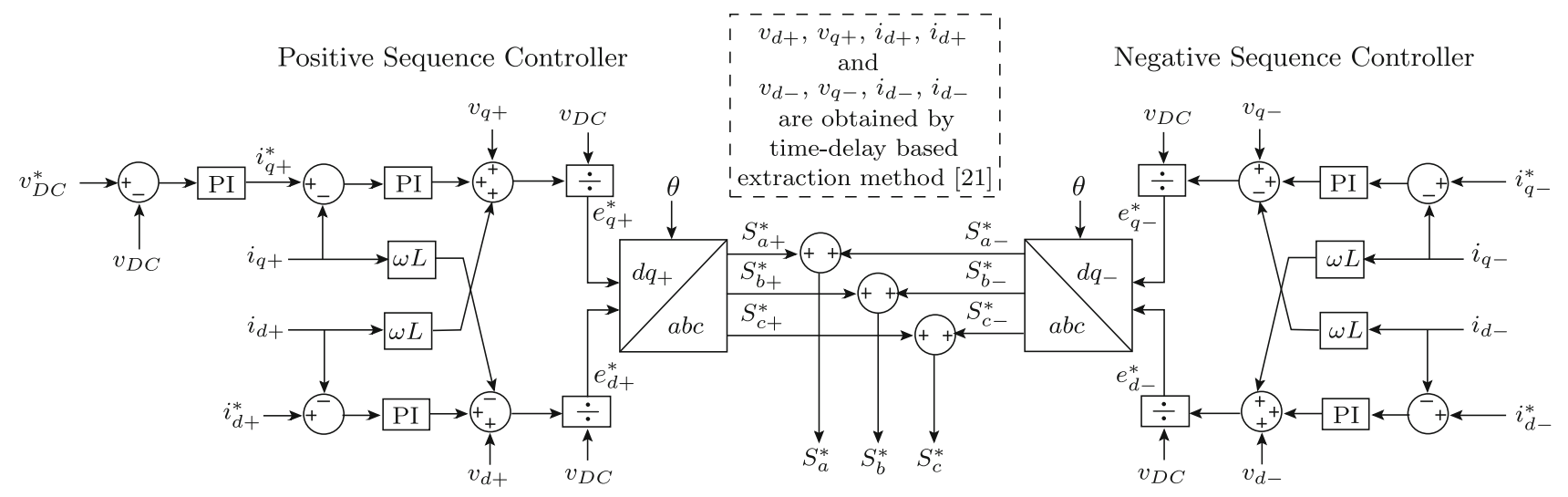

Figure 15. STATCOM with a sequence based controller [21].

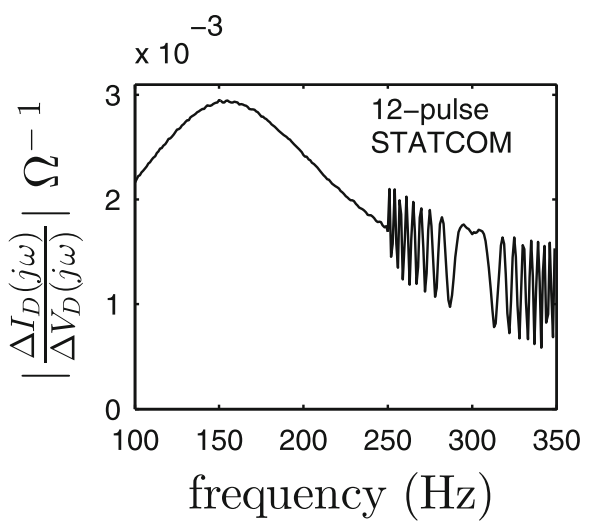

(a)

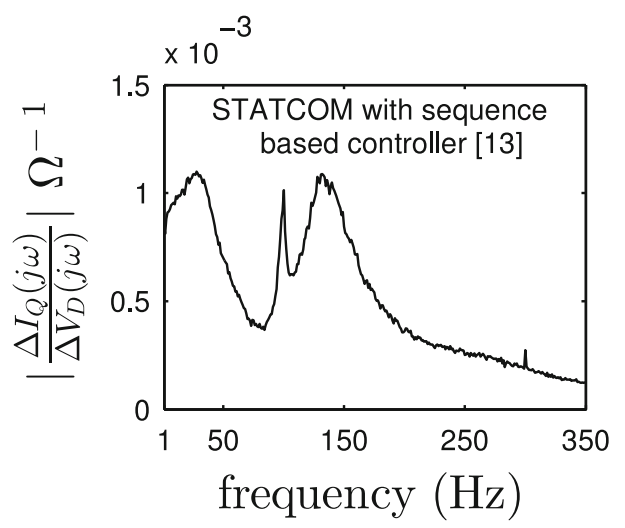

(b)

Figure 16. Frequency response of (a) 12-pulse STATCOM and (b) STATCOM with a sequence-based controller, obtained from a DQbased scan.

separating out the positive and negative sequence components, and having separate controller channels to control these components individually [13]. This is a useful feature for preventing unbalanced over-currents during faults. The transient positive and negative sequence components may be extracted by using notch-filters [21] or time-delays [22]. The structure of the controller is shown in figure 15 , where the subscripts ' + ' and ' - ' refer to the positive and negative sequence variables. The implementation of the controller using positive and negative sequence components will lead to a time-variant model in the DQ variables. Therefore, distortions are expected in the DQ based frequency scans.

The frequency responses obtained from the DQ based frequency scans $(1-350 \mathrm{~Hz})$ of a 12-pulse STATCOM and a PWM-switched STATCOM that uses a sequence based controller are shown in figure 16. The responses are not as smooth as those in figure 11. As expected, there is a significant distortion in the case of the 12-pulse STATCOM above $250 \mathrm{~Hz}$.

\section{Dynamic phasors [5]}

The $k^{\text {th }}$ Fourier coefficient or "Dynamic Phasor" $\langle x\rangle_{k}$ corresponding to a signal $x(\tau)$ is defined as follows.

$$
\langle x\rangle_{k}(t)=\frac{1}{T} \int_{t-T}^{t} x(\tau) e^{-j k \omega_{o} \tau} d \tau
$$

$x(\tau)$ can then be represented in terms of complex Fourier coefficients over a window length ' $T$ ' as follows:

$$
x(\tau)=\sum_{k=-\infty}^{\infty}\langle x\rangle_{k}(t) e^{j k \omega_{0} \tau} \quad \tau \in[t-T, t)
$$

where $\omega_{o}=\frac{2 \pi}{T}$. Usually, $\omega_{o}$ corresponds to the nominal supply frequency in $\mathrm{rad} / \mathrm{s}$.

The important properties of dynamic phasors are as follows.

(1) Derivative of the dynamic phasor is given by the following equation. 


$$
\frac{d\langle x\rangle_{k}}{d t}=\left\langle\frac{d x}{d t}\right\rangle_{k}-j k \omega_{0}\langle x\rangle_{k}
$$

This may be re-written as follows, by separating out the real and imaginary terms.

$$
\begin{aligned}
\frac{d\langle x\rangle_{k}^{r e}}{d t} & =\left\langle\frac{d x}{d t}\right\rangle_{k}^{r e}+k \omega_{0}\langle x\rangle_{k}^{i m} \\
\frac{d\langle x\rangle_{k}^{i m}}{d t} & =\left\langle\frac{d x}{d t}\right\rangle_{k}^{i m}-k \omega_{0}\langle x\rangle_{k}^{r e}
\end{aligned}
$$

(2) The dynamic phasor of the product of two signals $u(\tau)$ and $v(\tau)$ can be obtained by the discrete convolution of the corresponding dynamic phasors as follows:

$$
\langle u v\rangle_{k}=\sum_{l=-\infty}^{\infty}\langle u\rangle_{k-l}\langle v\rangle_{l}
$$

(3) If $x(\tau)$ is real, then $\langle x\rangle_{-l}=\langle x\rangle_{l}^{*}$

For time-periodic systems, dynamic phasors are constant in the steady state. The dynamic model of a system in dynamic phasor variables, $\langle x\rangle_{k}$, can be obtained from the original model, which is in terms $x$, using (9) [5]. Equation (11) is useful if multiplicative switching functions which are time-periodic in steady state are present in the original model. The presence of these multiplicative terms causes coupling between the dynamic phasors corresponding to different $k$. The dynamic phasor models of timeperiodic PES are time-invariant and lend themselves easily to conventional stability analysis [14].

\subsection{Example of a dynamic phasor model}

Consider a time-periodic system (with period $T=\frac{2 \pi}{\omega_{s}}$ ) represented by the equation:

$$
\frac{d x(t)}{d t}=\left(-10+\omega_{s} \cos \left(\omega_{s} t\right)\right) x(t)
$$

The solution of this equation is given below,

$$
x(t)=e^{\left(-10 t+\sin \left(\omega_{s} t\right)\right)} x(0) .
$$

This solution can be checked by putting $x(t)$ in Eq. (12).

The dynamic phasor model of the system given in (12) is obtained using (9) as follows:

$$
\begin{aligned}
\frac{d\langle x\rangle_{k}}{d t}= & -\left(10+j k \omega_{s}\right)\langle x\rangle_{k}+\omega_{s} \sum_{l=-\infty}^{\infty}\left\langle\cos \left(\omega_{s} t\right)\right\rangle_{l}\langle x(t)\rangle_{k-l} \\
= & -\left(10+j k \omega_{s}\right)\langle x\rangle_{k}+\omega_{s}\left\langle\cos \left(\omega_{s} t\right)\right\rangle_{1}\langle x(t)\rangle_{k-1} \\
& +\omega_{s}\left\langle\cos \left(\omega_{s} t\right)\right\rangle_{-1}\langle x(t)\rangle_{k+1} .
\end{aligned}
$$

There is a coupling between $\langle x\rangle_{k},\langle x\rangle_{k-1}$ and $\langle x\rangle_{k+1}$. This results in an infinite-dimensional state-space model. In practice, a limited number of dynamic phasors are considered to model the system and the number of dynamic phasors depends on the bandwidth of transient of interest. Considering real and imaginary parts of dynamic phasors as statevariables, the state-space model is obtained as given in (15).

$$
\langle X\rangle=A\langle X\rangle
$$

where $\langle X\rangle$ is the state vector that consists of dc and real and imaginary parts of dynamic phasors. The value of $\left\langle\cos \left(\omega_{s} t\right)\right\rangle_{1}$ and $\left\langle\cos \left(\omega_{s} t\right)\right\rangle_{-1}$ are constant which results in constant state matrix ' $A$ '. Hence, the developed dynamic phasor model is time-invariant.

The solution of the dynamic phasor model is

$$
\langle X\rangle(t)=e^{A\left(t-t_{0}\right)}\langle X\rangle\left(t_{0}\right)
$$

where $\langle X\rangle\left(t_{0}\right)$ is the value of all dynamic phasor components at $t=t_{0}$.

3.1a Reconstruction of signal $x(t)$ using dynamic phasors: Reconstruction of $x(t)$ using its dynamic phasors is possible using following delayed reconstruction formula [23]:

$$
x(\tau)=\sum_{k=-\infty}^{\infty}\langle x\rangle_{k}(t) e^{j k \omega_{0} \tau} \quad \text { where } \tau=\mathrm{t}-\epsilon, 0<\epsilon<\mathrm{T} .
$$

Now we reconstruct $x(t)$ from its dynamic phasors and the accuracy of reconstruction is checked with the exact solution of $x(t)$ as given in (13). Three different values of $\epsilon=T$, $T / 2$ and $T / 4$ are used. For initial condition, $t_{0}=T$ is considered and $\langle X\rangle(T)$ is numerically evaluated from the actual response of $x(t)$ using $(7):\langle x\rangle_{0}(T)=1.1796,\langle x\rangle_{1}^{r e}(T)=$ 0.0087, $\langle x\rangle_{1}^{i m}(T)=-0.5512, \quad\langle x\rangle_{2}^{r e}(T)=-0.1344$, $\langle x\rangle_{2}^{i m}(T)=-0.0177, \quad\langle x\rangle_{3}^{r e}(T)=-0.0042, \quad\langle x\rangle_{3}^{i m}(T)=$ 0.0126 and so on.

3.1b Case 1: $k= \pm 1$ and 0 : The dynamic phasor model is obtained considering fundamental and DC dynamic phasor (other dynamic phasors are neglected) using (14) as follows:

$$
\frac{d}{d t}\left[\begin{array}{c}
\langle x\rangle_{0} \\
\langle x\rangle_{1}^{r e} \\
\langle x\rangle_{1}^{i m}
\end{array}\right]=\left[\begin{array}{ccc}
-10 & \omega_{s} & 0 \\
0.5 \omega_{s} & -10 & \omega_{s} \\
0 & -\omega_{s} & -10
\end{array}\right]\left[\begin{array}{c}
\langle x\rangle_{0} \\
\langle x\rangle_{1}^{r e} \\
\langle x\rangle_{1}^{i m}
\end{array}\right] .
$$

Eigen-values of this system are -10 and $-10 \pm j 222.14$. The solution of the dynamic phasors is obtained using (16). Signal $x(t)$ is reconstructed using (17) with different values of $\epsilon$ and shown in figure 17. There is an error in the reconstructed signal and the exact signal. Note that for $\epsilon=$ $T, T / 2$ and $T / 4$, the reconstructed signal is obtained from $t=0, t=T / 2$ and $3 T 4$, respectively.

Case 2: $k= \pm 3, \pm 2, \pm 1$ and $0 \quad$ The dynamic phasor model is given below: 


$$
\frac{d}{d t}\left[\begin{array}{c}
\langle x\rangle_{0} \\
\langle x\rangle_{1}^{r e} \\
\langle x\rangle_{1}^{i m} \\
\langle x\rangle_{2}^{r e} \\
\langle x\rangle_{2}^{i m} \\
\langle x\rangle_{3}^{r e} \\
\langle x\rangle_{3}^{i m}
\end{array}\right]=\left[\begin{array}{ccccccc}
-10 & \omega_{s} & 0 & 0 & 0 & 0 & 0 \\
0.5 \omega_{s} & -10 & \omega_{s} & 0.5 \omega_{s} & 0 & 0 & 0 \\
0 & -\omega_{s} & -10 & 0 & 0.5 \omega_{s} & 0 & 0 \\
0 & 0.5 \omega_{s} & 0 & -10 & 2 \omega_{s} & 0.5 \omega_{s} & 0 \\
0 & 0 & 0.5 \omega_{s} & -2 \omega_{s} & -10 & 0 & 0.5 \omega_{s} \\
0 & 0 & 0 & 0.5 \omega_{s} & 0 & -10 & 3 \omega_{s} \\
0 & 0 & 0 & 0 & 0.5 \omega_{s} & -3 \omega_{s} & -10
\end{array}\right]\left[\begin{array}{c}
\langle x\rangle_{0} \\
\langle x\rangle_{1}^{r e} \\
\langle x\rangle_{1}^{i m} \\
\langle x\rangle_{2}^{r e} \\
\langle x\rangle_{2}^{i m} \\
\langle x\rangle_{3}^{r e} \\
\langle x\rangle_{3}^{i m}
\end{array}\right] .
$$

The eigen-values of the above system are $-10 \pm j 313.67$, $-10 \pm j 643.45,-10 \pm j 849.28$ and -10 . Similarly signal is reconstructed using dynamic phasors and shown in figure 18. There is a close match between the reconstructed signal and exact solution of $x(t)$ for $\epsilon=T / 2$ and $T / 4$.

Similarly, the eigen-values obtained by considering dynamic phasors of $k=5,4,3,2,1$ and 0 are $-10 \pm j 314.16, \quad-10 \pm j 628.33, \quad-10 \pm j 941.99$, $-10 \pm j 1271.8,-10 \pm j 1477.6$ and -10 .

\section{Remarks}

1. Reconstruction with $\epsilon=T$ gives inaccurate results even if many dynamic phasors are considered.

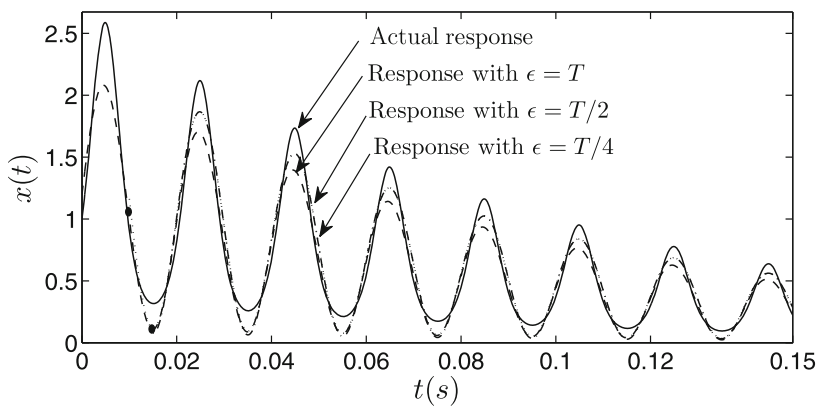

Figure 17. Plot of time domain signal, $x(t)$ for exact and dynamic phasor $(k=1$ and 0$)$ solutions.

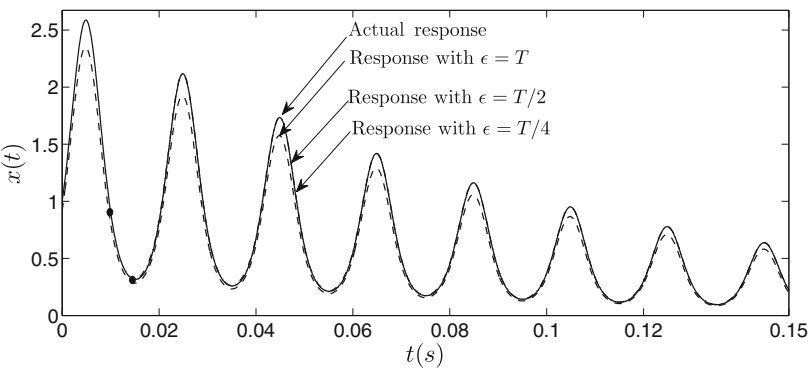

Figure 18. Plot of time domain signal $x(t)$ for exact and dynamic phasor $(k=3,2,1$ and 0 ) solutions (responses with $\epsilon=T / 2$ and $T / 4$ completely overlapped with the actual response).
2. With $\epsilon<T$, the reconstruction becomes more accurate as $k$ is increased.

3. The low-frequency eigen-values of the dynamic phasor model seem to converge as $k$ is increased.

4. The dynamic phasor model shows that the system is stable. This is consistent with the actual system response.

\section{Dynamic phasor based frequency scanning procedure}

In the extraction of the frequency response in the dynamic phasor variables we encounter several issues that are not encountered in phase/sequence or DQ based scanning. These issues are discussed here.

(1) Theoretically, there are infinite dynamic phasors $(k=-\infty$ to $\infty)$ for a signal injected or observed in the time-domain simulation of the system. For practical systems, consideration of a limited number of dynamic phasors is usually adequate for stability analysis.

For a single-phase system, even if a few phasors are considered, say $(k=0,1)$, then the admittance is in the form of a matrix transfer function as follows:

$$
\left[\begin{array}{c}
\langle\Delta I\rangle_{0}(j \omega) \\
\langle\Delta I\rangle_{1}^{r e}(j \omega) \\
\langle\Delta I\rangle_{1}^{i m}(j \omega)
\end{array}\right]=\left[\begin{array}{lll}
G_{11} & G_{12} & G_{13} \\
G_{21} & G_{22} & G_{23} \\
G_{31} & G_{32} & G_{33}
\end{array}\right]\left[\begin{array}{c}
\langle\Delta V\rangle_{0}(j \omega) \\
\langle\Delta V\rangle_{1}^{r e}(j \omega) \\
\langle\Delta V\rangle_{1}^{i m}(j \omega)
\end{array}\right] .
$$

Notation: $\langle\Delta I\rangle_{1}^{r e}(j \omega)$ is the frequency domain representation of $\langle\Delta i\rangle_{1}^{r e}$, which is the real part of the dynamic phasor of $\Delta i(t) .\langle\Delta I\rangle_{1}^{i m}(j \omega)$ is the frequency domain representation of $\langle\Delta i\rangle_{1}^{i m}$, which is the imaginary part of the dynamic phasor of $\Delta i(t)$. Note that both $\langle\Delta i\rangle_{1}^{r e}$ and $\langle\Delta i\rangle_{1}^{i m}$ are real signals, whereas $\langle\Delta I\rangle_{1}^{r e}(j \omega)$ and $\langle\Delta I\rangle_{1}^{i m}(j \omega)$ are complex numbers.

Since the time-domain simulation model is generally not in dynamic phasor variables, we have to use a discrete-time approximation of (7) to evaluate $\langle\Delta i\rangle_{k}$ and $\langle\Delta v\rangle_{k}$ from $\Delta i(t)$ 
and $\Delta v(t)$, respectively. FFT algorithm is then used to obtain $\langle\Delta I\rangle_{k}^{r e}(j \omega),\langle\Delta I\rangle_{k}^{i m}(j \omega),\langle\Delta V\rangle_{k}^{r e}(j \omega)$ and $\langle\Delta V\rangle_{k}^{i m}(j \omega)$.

(2) It is not possible to exclusively inject one dynamic phasor at a time. If the input signal is $u(t)=e^{j \omega_{i} t}$, then in terms of dynamic phasor variables, it implies a simultaneous injection of $\langle u\rangle_{0}$ at the frequency $\omega_{i},\langle u\rangle_{1}=\langle u\rangle_{1}^{r e}+j\langle u\rangle_{1}^{i m}$ at $\omega_{i}-\omega_{o},\langle u\rangle_{2}=\langle u\rangle_{2}^{r e}+j\langle u\rangle_{2}^{i m}$ at $\omega_{i}-2 \omega_{o}$, and so on.

If the underlying system is time-invariant in the dynamic phasor variables, then the dynamic phasor of the output signal $y(t)$ will contain these frequencies as well. Moreover, one can attribute all the output dynamic phasors at the frequency $\omega_{i}$ to the input $\langle u\rangle_{0}$, the dynamic phasors at the frequency $\omega_{i}-\omega_{o}$ to the input $\langle u\rangle_{1}$ and so on.

In other words, an injection of $u(t)$ at the frequency $\omega_{i}$ will yield the following frequency responses in the steady state:

$$
\begin{gathered}
\frac{\langle Y\rangle_{0}(s)}{\langle U\rangle_{0}(s)}, \frac{\langle Y\rangle_{1}(s)}{\langle U\rangle_{0}(s)}, \frac{\langle Y\rangle_{2}(s)}{\langle U\rangle_{0}(s)}, \ldots . \text { at } s=j \omega_{i} \\
\frac{\langle Y\rangle_{0}(s)}{\langle U\rangle_{1}(s)}, \frac{\langle Y\rangle_{1}(s)}{\langle U\rangle_{1}(s)}, \frac{\langle Y\rangle_{2}(s)}{\langle U\rangle_{1}(s)}, \ldots . \text { at } s=j\left(\omega_{i}-\omega_{0}\right) \\
\frac{\langle Y\rangle_{0}(s)}{\langle U\rangle_{2}(s)}, \frac{\langle Y\rangle_{1}(s)}{\langle U\rangle_{2}(s)}, \frac{\langle Y\rangle_{2}(s)}{\langle U\rangle_{2}(s)}, \ldots . \text { at } s=j\left(\omega_{i}-2 \omega_{0}\right) \\
\frac{\langle Y\rangle_{0}(s)}{\langle U\rangle_{n}(s)}, \frac{\langle Y\rangle_{1}(s)}{\langle U\rangle_{n}(s)}, \frac{\langle Y\rangle_{2}(s)}{\langle U\rangle_{n}(s)}, \ldots . \text { at } s=j\left(\omega_{i}-n \omega_{0}\right) .
\end{gathered}
$$

Similarly, for the input $u(t)=e^{-j \omega_{i} t}$,

$$
\begin{aligned}
& \frac{\langle Y\rangle_{0}(s)}{\langle U\rangle_{0}(s)}, \frac{\langle Y\rangle_{1}(s)}{\langle U\rangle_{0}(s)}, \frac{\langle Y\rangle_{2}(s)}{\langle U\rangle_{0}(s)}, \ldots \text { at } s=-j \omega_{i} \\
& \frac{\langle Y\rangle_{0}(s)}{\langle U\rangle_{1}(s)}, \frac{\langle Y\rangle_{1}(s)}{\langle U\rangle_{1}(s)}, \frac{\langle Y\rangle_{2}(s)}{\langle U\rangle_{1}(s)}, \ldots \text { at } s=-j\left(\omega_{i}+\omega_{0}\right) \\
& \frac{\langle Y\rangle_{0}(s)}{\langle U\rangle_{2}(s)}, \frac{\langle Y\rangle_{1}(s)}{\langle U\rangle_{2}(s)}, \frac{\langle Y\rangle_{2}(s)}{\langle U\rangle_{2}(s)}, \ldots \text { at } s=-j\left(\omega_{i}+2 \omega_{0}\right) \\
& \ldots . \\
& \frac{\langle Y\rangle_{0}(s)}{\langle U\rangle_{n}(s)}, \frac{\langle Y\rangle_{1}(s)}{\langle U\rangle_{n}(s)}, \frac{\langle Y\rangle_{2}(s)}{\langle U\rangle_{n}(s)}, \ldots . \text { at } s=-j\left(\omega_{i}+n \omega_{0}\right) .
\end{aligned}
$$

Note that for $k \neq 0$,

$$
\begin{aligned}
\langle U\rangle_{k}(s) & =\langle U\rangle_{k}^{r e}(s)+j\langle U\rangle_{k}^{i m}(s) \\
\langle Y\rangle_{k}(s) & =\langle Y\rangle_{k}^{r e}(s)+j\langle Y\rangle_{k}^{i m}(s) .
\end{aligned}
$$

Since the real and imaginary components of input and output dynamic phasors are real signals

$$
\begin{array}{r}
\langle U\rangle_{k}^{r e}\left(j \omega_{i}\right)=\langle U\rangle_{k}^{r e *}\left(-j \omega_{i}\right),\langle U\rangle_{k}^{i m}\left(j \omega_{i}\right)=\langle U\rangle_{k}^{i m^{*}}\left(-j \omega_{i}\right) \\
\langle Y\rangle_{k}^{r e}\left(j \omega_{i}\right)=\langle Y\rangle_{k}^{r e *}\left(-j \omega_{i}\right),\langle Y\rangle_{k}^{i m}\left(j \omega_{i}\right)=\langle Y\rangle_{k}^{i m^{*}}\left(-j \omega_{i}\right)
\end{array}
$$

where ' $*$ ' represents the complex conjugate operation. To obtain the frequency responses of individual real and imaginary components, we can use the following relationships.

$$
\text { If } \begin{aligned}
\mathrm{M}=\frac{\langle\mathrm{Y}\rangle_{\mathrm{k}}(\mathrm{j} \omega)}{\langle\mathrm{U}\rangle_{\mathrm{m}}(\mathrm{j} \omega)} \text { and } \mathrm{N}=\frac{\langle\mathrm{Y}\rangle_{\mathrm{k}}(-\mathrm{j} \omega)}{\langle\mathrm{U}\rangle_{\mathrm{m}}(-\mathrm{j} \omega)} \text { then } \\
{\left[\begin{array}{c}
\langle Y\rangle_{k}^{r e}(j \omega) \\
\langle Y\rangle_{k}^{i m}(j \omega)
\end{array}\right]=\left[\begin{array}{cc}
1 & j \\
1 & -j
\end{array}\right]^{-1}\left[\begin{array}{cc}
M & j M \\
N^{*} & -j N^{*}
\end{array}\right]\left[\begin{array}{c}
\langle U\rangle_{m}^{r e}(j \omega) \\
\langle U\rangle_{m}^{i m}(j \omega)
\end{array}\right] . }
\end{aligned}
$$

Although the analysis presented so far uses complex input signals $e^{j \omega_{i} t}$ and $e^{-j \omega_{i} t}$, actual injection is in terms of real sinusoidal and cosinusoidal (i.e., phase shifted by $\frac{\pi}{2}$ ) signals, from which we can obtain the required responses, using the well known relationships between these functions.

(3) If all components of the matrix transfer function corresponding to the dynamic phasors from $k=0$ to $n$ in a frequency range $0-\omega_{\max }$ are desired, then $\omega_{i}$ of the input signal has to be varied from 0 to $\left(\omega_{\max }+n \omega_{0}\right)$.

(4) If a multi-sine signal is injected, then it is important to avoid overlap of frequencies in order to correctly identify all transfer functions. Therefore, to obtain the frequency response from, say, 0 to $3 \omega_{o}$, we need to carry out three scans: (i) from 0 to $\omega_{o}$, (ii) from $\omega_{o}$ to $2 \omega_{o}$ and (iii) from $2 \omega_{o}$ to $3 \omega_{o}$. For threephase systems, the injection will have to be done one phase or one sequence component at a time. It is not possible to obtain the frequency response at the exact multiples of the fundamental frequency, as dynamic phasor components at these frequencies cannot be recovered (they are zero). Therefore, one may infer these by interpolation.

The complete procedure for scanning based on dynamic phasor variables is depicted in figure 19 .

Complexities involved in the dynamic phasor based frequency scanning as illustrated using the example of a 12-pulse STATCOM. A sinusoidal signal $u(t)=\sin (2 \pi 20 t)$ at a single frequency of $20 \mathrm{~Hz}$ is injected in the phase ' $a$ ' of the STATCOM (no injection in phase ' $b$ ' and 'c'). The frequency components present in the dynamic phasor components of input voltage are shown in figure 20. As expected, the $20-\mathrm{Hz}$ component is present in $\left\langle\Delta V_{a}\right\rangle_{0}, 30$ and $70 \mathrm{~Hz}$ components are present $\left\langle\Delta V_{a}\right\rangle_{1}, 80$ and $120 \mathrm{~Hz}$ are present in $\left\langle\Delta V_{a}\right\rangle_{2}$ and 130 and $170 \mathrm{~Hz}$ are present in $\left\langle\Delta V_{a}\right\rangle_{3}$.

The dynamic phasors extracted using output currents are shown in figure 20 . In $\left\langle\Delta I_{a}\right\rangle_{0}$, the frequency component at $20 \mathrm{~Hz}$ is generated due to the input $\left\langle\Delta V_{a}\right\rangle_{0}$ and 80 and $120 \mathrm{~Hz}$ components are generated due to $\left\langle\Delta V_{a}\right\rangle_{2}$. Similarly, the presence of different frequency components present in the other current dynamic phasors can be explained.

\section{Examples of dynamic phasor based scanning}

\section{$5.1 T C S C$}

Consider a TCSC connected to a $50 \mathrm{~Hz}$ current source as shown in figure 21(a). The parameters of the TCSC are as 
1)

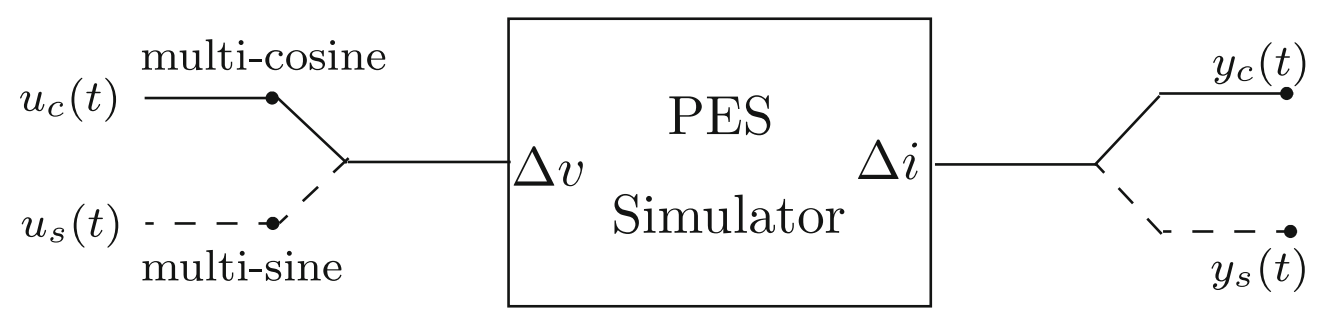

2)
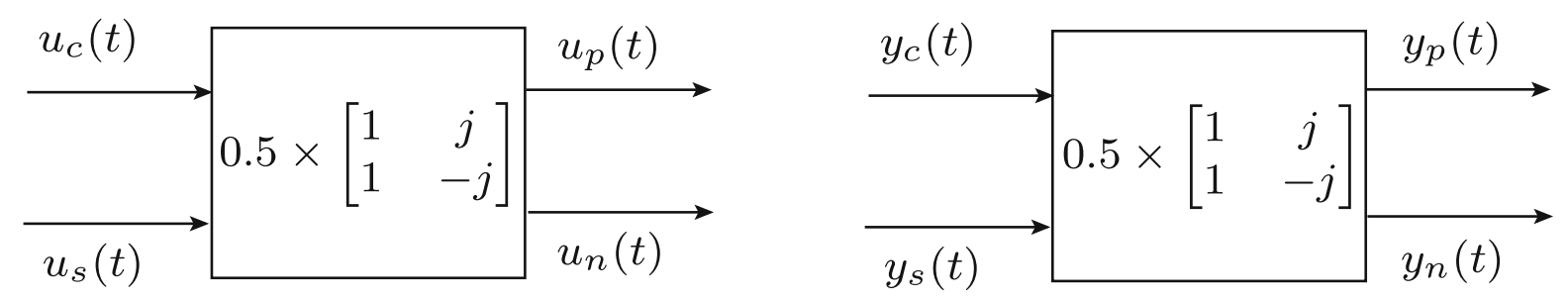

3)

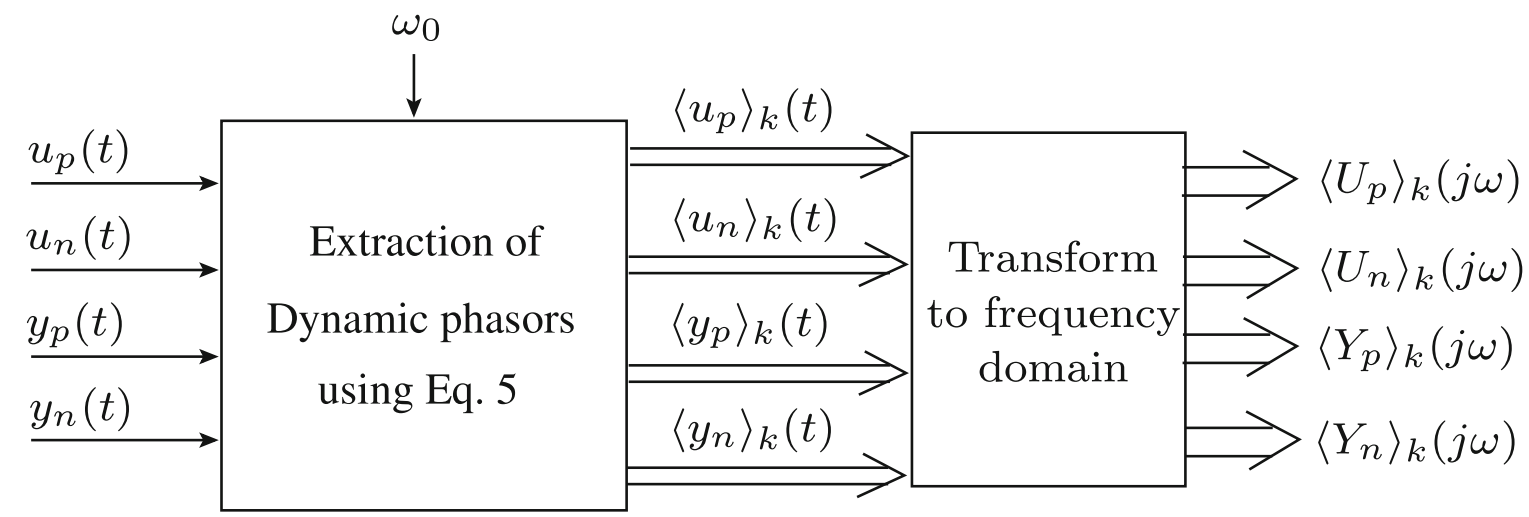

4) For each $l=0,1,2, \ldots k$ and $m=0,1,2, \ldots k$
$\left\langle U_{p}\right\rangle_{l}(j \omega)$
$\left\langle Y_{p}\right\rangle_{m}\left(\overrightarrow{j \omega)} \stackrel{\downarrow}{\bullet} \underset{H_{m l}}{\longrightarrow}\left(j\left(\omega+l \omega_{0}\right)\right)\right.$

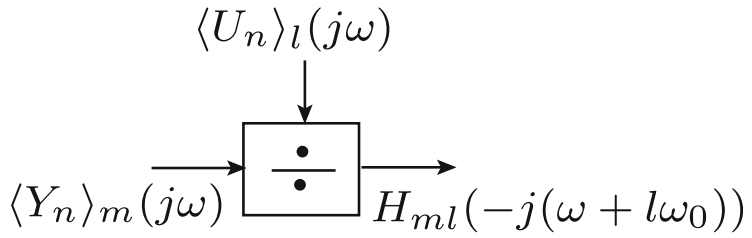

5) Separation of frequency response w.r.t real and imaginary components:

$$
\left[\begin{array}{c}
\langle Y\rangle_{m}^{r e}(j \omega) \\
\langle Y\rangle_{m}^{i m}(j \omega)
\end{array}\right]=\left[\begin{array}{cc}
1 & j \\
1 & -j
\end{array}\right]^{-1}\left[\begin{array}{cc}
H_{m l}(j \omega) & j H_{m l}(j \omega) \\
H_{m l}(-j \omega)^{*} & -j H_{m l}(-j \omega)^{*}
\end{array}\right]\left[\begin{array}{c}
\langle U\rangle_{l}^{r e}(j \omega) \\
\langle U\rangle_{l}^{i m}(j \omega)
\end{array}\right]
$$

Figure 19. Frequency scanning technique using dynamic phasor variables.

follows [24]: $L=12.4 \mathrm{mH}, C=82 \mu \mathrm{F}$, and the rms value of the current source is $1 \mathrm{kA}$. The TCSC operates at a firing delay angle of $63^{\circ}$. The firing pulses are generated with respect to the positive zero crossing of the line current.

To obtain the frequency scan, a multi-sine current $\Delta i$ is injected as shown in figure 21(a). Note that the simulation includes the switching action of the thyristors and the firing
Figure 20. Frequencies seen in the dynamic phasor components of the voltage of phase ' $\mathrm{a}$ ' when a sinusoidal voltage $u(t)=$ $\sin (2 \pi 20 t)$ which contains a single frequency, $20 \mathrm{~Hz}$, is injected in phase 'a' of a 12-pulse STATCOM.

angle synchronization with the line current. The multi-sine parameters are $a=0.001 \mathrm{kA}$ and $f_{d}=1 \mathrm{~Hz}$. The frequency scanning range is from 1 to $200 \mathrm{~Hz}$. 


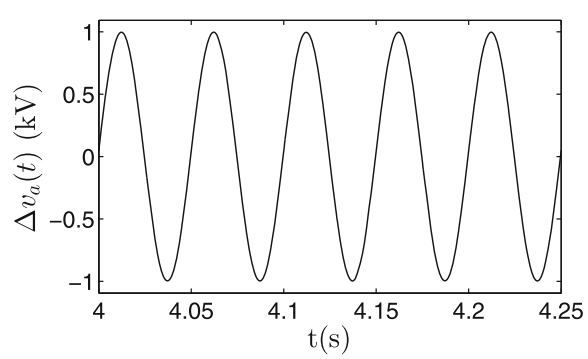

(a)

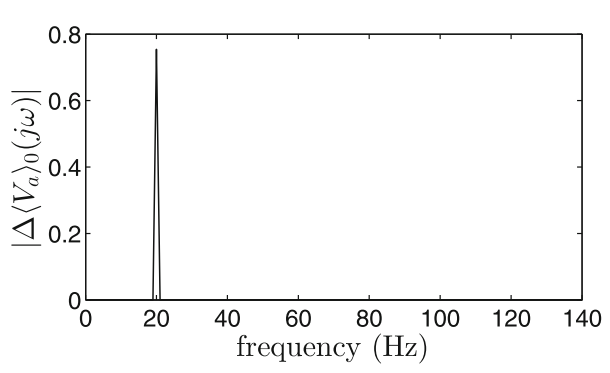

(c)

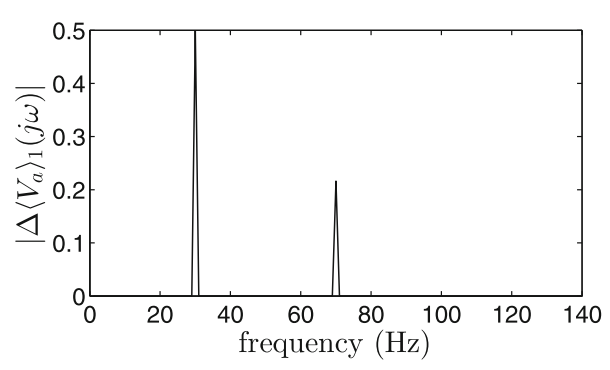

(e)

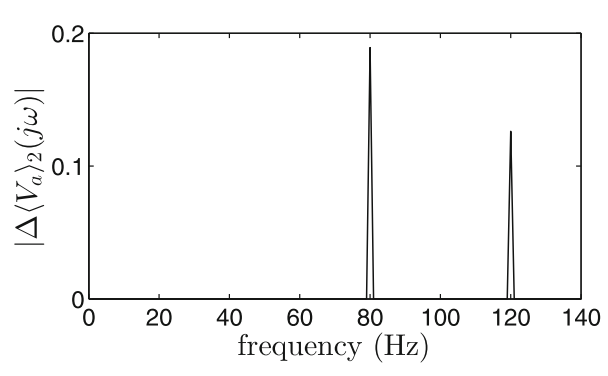

(g)

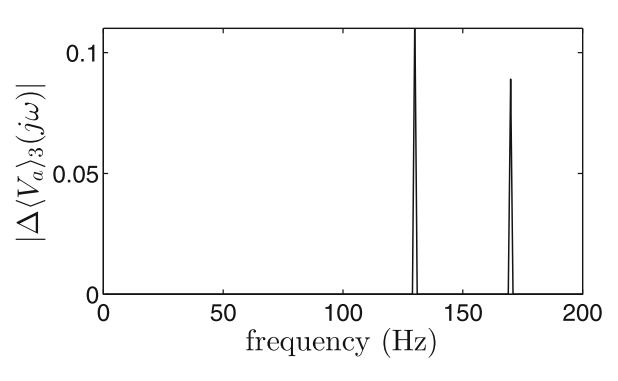

(i)

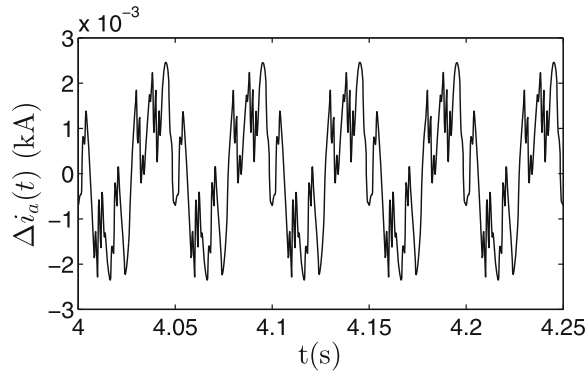

(b)

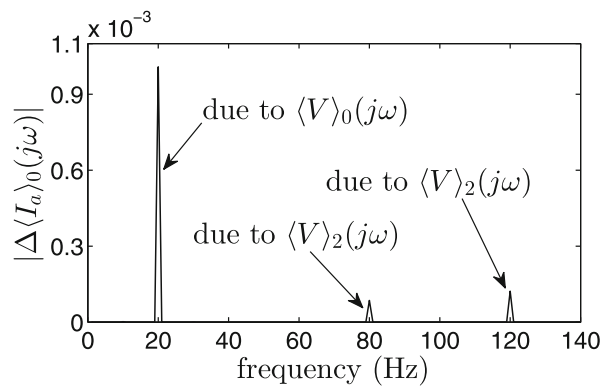

(d)

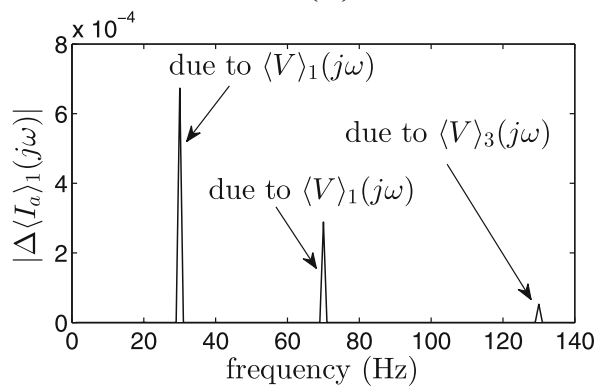

(f)

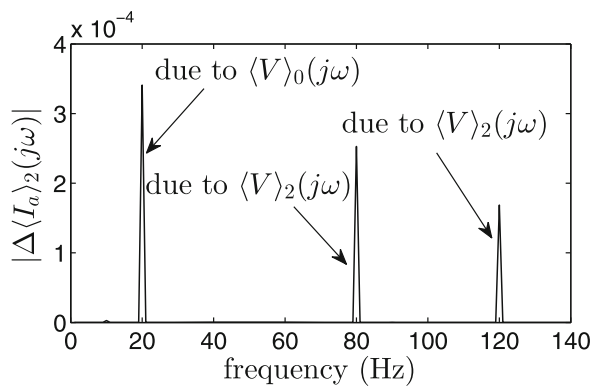

(h)

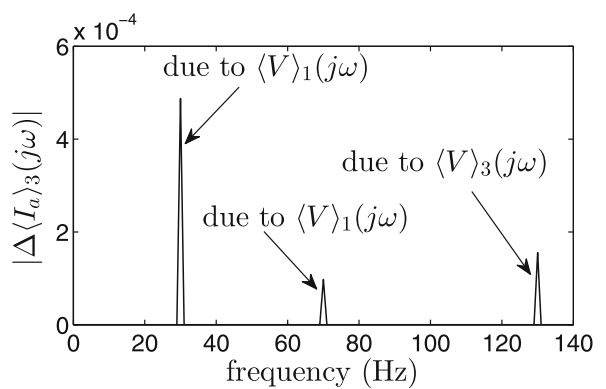

(j) 


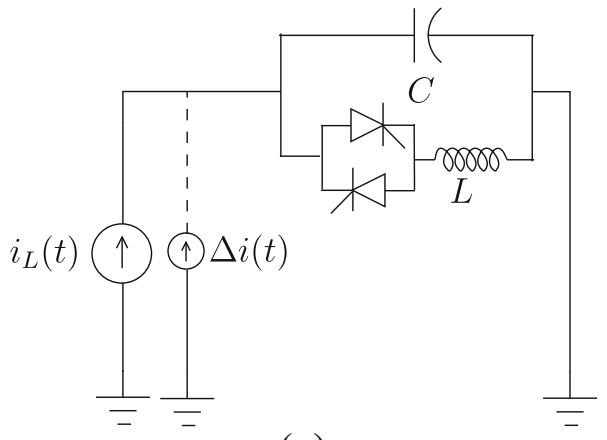

(a)

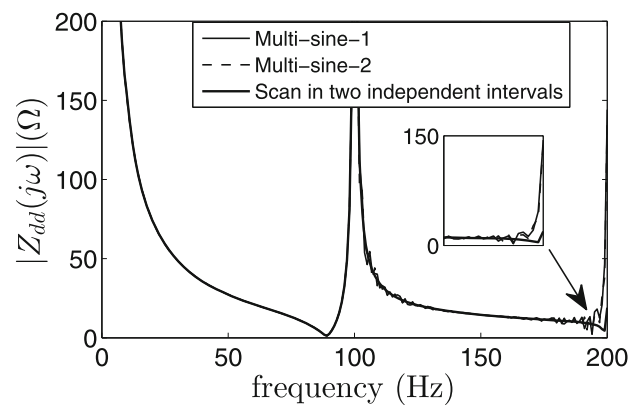

(b)

Figure 21. (a) Frequency scanning set-up for a TCSC and (b) frequency response of TCSC obtained by DQ based scanning (Quiescent delay angle, $\left.\alpha_{0}=156^{\circ}\right)$.

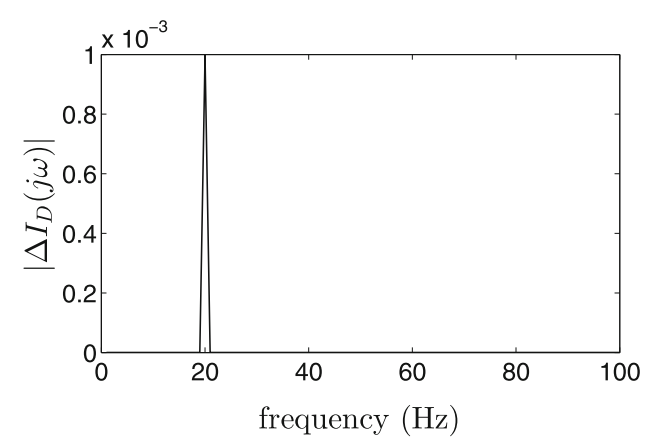

(a)

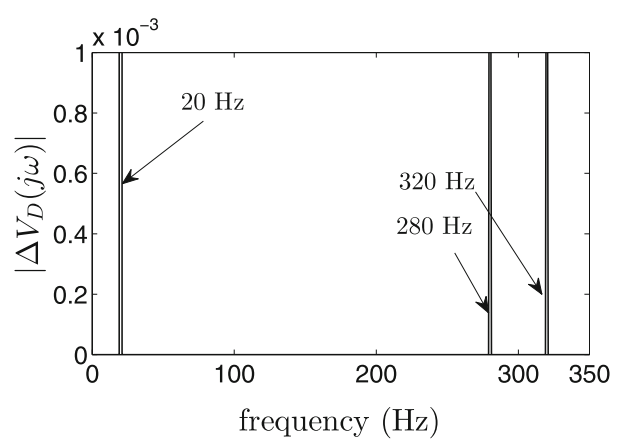

(b)

Figure 22. TCSC: frequencies seen in the D-component of the voltage when the inject current, $\Delta i_{D}$ is a sinusoidal signal at a single frequency, $20 \mathrm{~Hz}$.

The frequency response of the TCSC using different multi-sine signals is shown in figure 21. Multi-sine-1 uses Schroeder's multi-sine signal-see Eq. (2). Multisine- 2 is generated by minimizing the overall magnitude of $u(t)$ (1) in the selected frequency range using a genetic algorithm based optimization tool in MATLAB [20]. The resulting frequency response shows distortions from $100 \mathrm{~Hz}$ to $200 \mathrm{~Hz}$ as shown in figure 21. The switching harmonic at $300 \mathrm{~Hz}$ (in the DQ frame) multiplicatively interacts with the frequency components between 100 and $200 \mathrm{~Hz}$ of the wide-band signal, to cause additional interfering components between 100 and $200 \mathrm{~Hz}$. This is also illustrated in figure 22, for a single frequency injection at $20 \mathrm{~Hz}$ in the input $\mathrm{D}$-channel. The $20-\mathrm{Hz}$ signal interacts with the $300-\mathrm{Hz}$ switching harmonic to cause components at $280 \mathrm{~Hz}$ and $320 \mathrm{~Hz}$. If two independent scans are carried out $(0-150 \mathrm{~Hz}$ and $150-200 \mathrm{~Hz})$, then the additional components in each scan fall outside the interval of that scan, and are easy to identify and discard. This results in a smoother overall scan, as shown in figure 21 . The scans have significant differences in the higher frequency range, which is indicative of the time-invariance of the system in the DQ variables due to the low order harmonics.

In the following sub-section, we obtain the dynamic phasor scans using the procedure presented in the previous section, and also compare them with those obtained using an approximate analytical model.

5.1a Approximate analytical model of a TCSC: The state equations representing TCSC (for one phase) are given by the following equations:

$$
\begin{gathered}
L \frac{d i}{d t}=q v_{c} \\
C \frac{d v_{c}}{d t}=i_{L}-i
\end{gathered}
$$

where $i_{L}$ represents the line current; $v_{c}$ and $i$ denote the voltage across the capacitor and the current through the inductor, respectively; ' $q$ ' is the switching function which depends on the thyristor states i.e., if a thyristor is on, $q=1$, and $q=0$ when a thyristor is off.

Using (9) and (10), the equations given in (22) are rewritten in terms of the dynamic phasors. The equations for the $k^{\text {th }}$ dynamic phasor are as follows: 

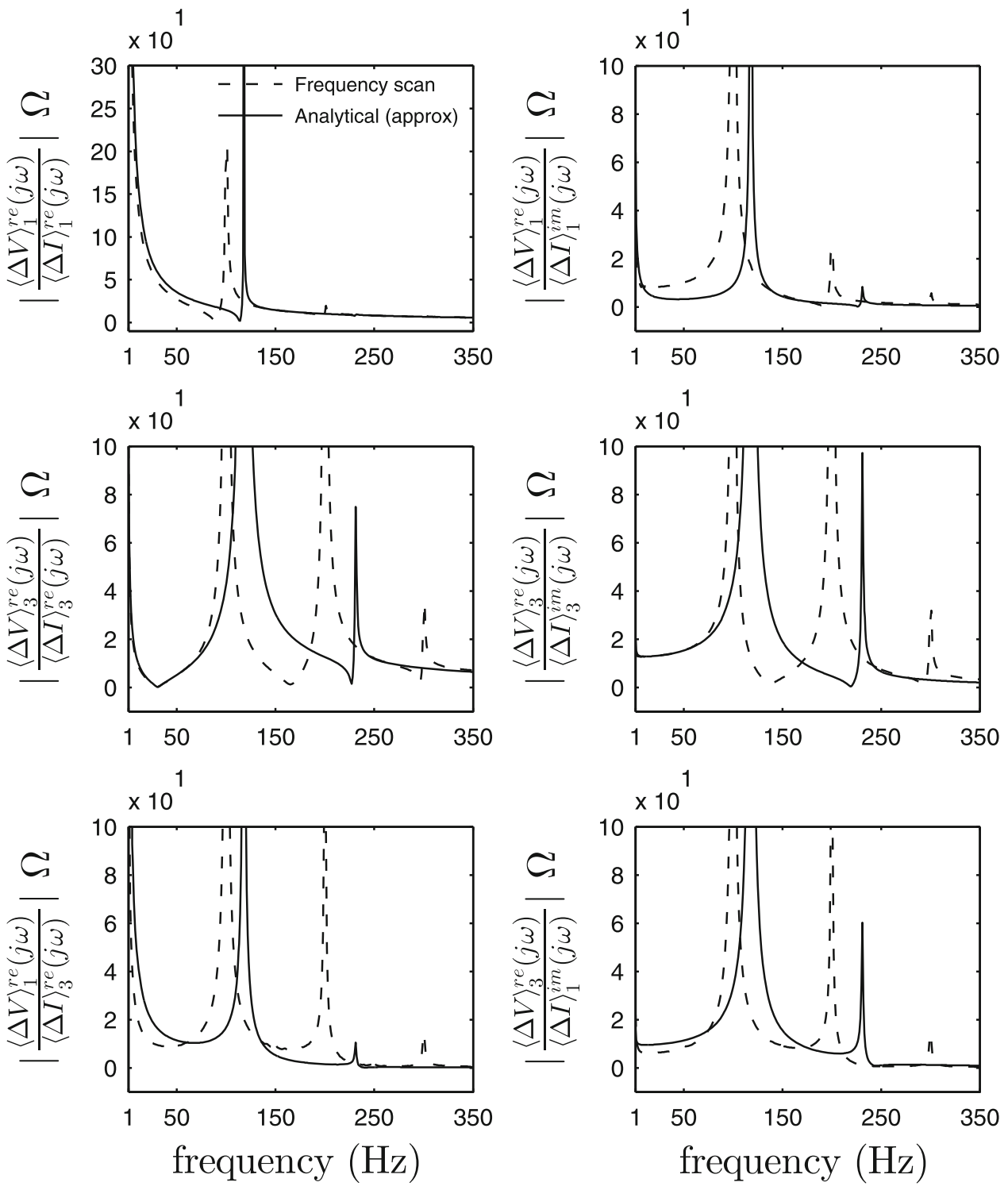

Figure 23. Frequency response of TCSC (dynamic phasor based) for one phase obtained analytically and by frequency scanning.

$$
\begin{aligned}
L \frac{d\langle i\rangle_{k}^{r e}}{d t} & =k \omega_{0}\langle i\rangle_{k}^{i m}+\left\langle q v_{c}\right\rangle_{k}^{r e} \\
L \frac{d\langle i\rangle_{k}^{i m}}{d t} & =-k \omega_{0}\langle i\rangle_{k}^{r e}+\left\langle q v_{c}\right\rangle_{k}^{i m} \\
C \frac{d\left\langle v_{c}\right\rangle_{k}^{r e}}{d t} & =k \omega_{0}\left\langle v_{c}\right\rangle_{k}^{i m}+\left\langle i_{L}\right\rangle_{k}^{r e}-\langle i\rangle_{k}^{r e} \\
C \frac{d\left\langle v_{c}\right\rangle_{k}^{i m}}{d t} & =-k \omega_{0}\left\langle v_{c}\right\rangle_{k}^{r e}+\left\langle i_{L}\right\rangle_{k}^{i m}-\langle i\rangle_{k}^{i m} .
\end{aligned}
$$

The term $\left\langle q v_{c}\right\rangle_{k}$ creates a coupling between dynamic phasors corresponding to different values of $k$ as a result of (11).

Since the turn-off instants of the thyristors are dependent on the circuit conditions, switching function ' $q$ ' may be obtained by iteratively solving transcendental equations [25], which makes the model complicated. In [5] and [25], it is assumed that the peak of the actual inductor current is symmetrical about its fundamental component, and $k$ is restricted to 1,3 and 5. Since low-frequency transients are of interest, the differential equations corresponding to $\langle i\rangle_{k}$ are converted to algebraic equations by replacing the derivative of $\langle i\rangle_{k}$ with zero. This results in an approximate low-frequency model having six states per phase for a constant firing angle delay. This model is linearized and the frequency responses may be obtained analytically.

5.1b Comparison of the frequency responses: The dynamic phasor based impedance obtained from the 

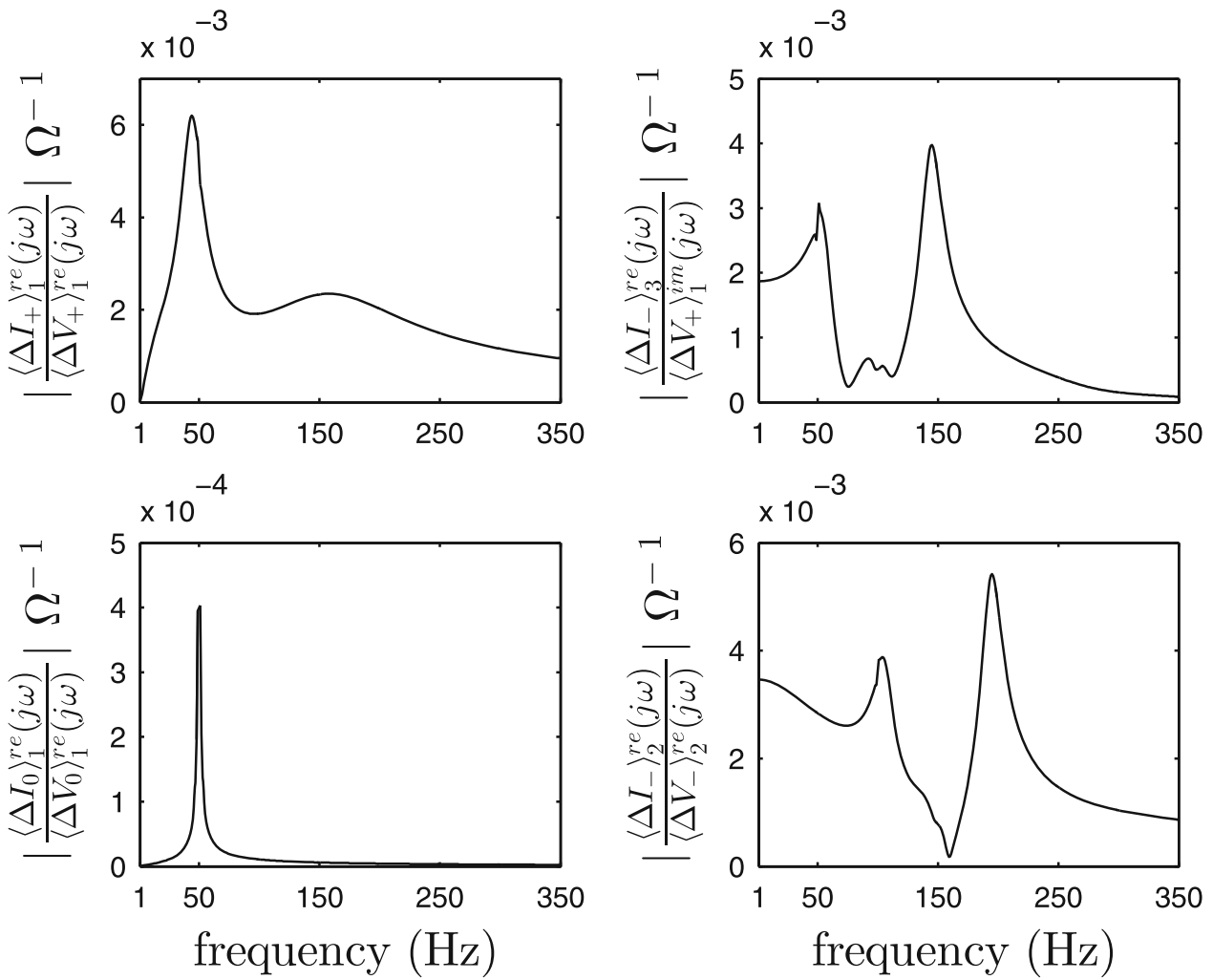

Figure 24. Frequency response obtained from a dynamic phasor based scan of a 12-pulse STATCOM.

analytical model and the one obtained from the simulation based scan is shown in figure 23. Only a few transfer function components are shown. At low frequencies both the responses are identical but are significantly different at high frequencies. This is expected, as the analytical model is an approximate one and is valid only at low frequencies. Note that the dynamic phasor scans are much smoother compared to the DQ based scan shown in figure 21 .

\subsection{STATCOM}

Dynamic phasor based scanning of the 12-pulse STATCOM and the PWM-switched STATCOM with a sequence controller (considered in section 2) is carried out. The multi-sine parameters are same as used earlier in the DQ based scan.

The frequency response of some of the elements of admittance matrix (in terms of sequence components of the dynamic phasors) is shown in figures 24 and 25 . The responses are smooth, indicating the time invariance property of the underlying model in dynamic phasor variables.

Table 1 presents different systems encountered in practice and the nature of various models. A time-domain signal $x(t)$ and its $k^{\text {th }}$ dynamic phasor $\langle x\rangle_{k}(t)$ are related in frequency domain as follows: $\langle X\rangle_{k}(s)=\frac{1}{T} \frac{\left(1-e^{-s T}\right)}{s} X\left(s+j k \omega_{o}\right)$.

\section{Grid interaction studies using dynamic phasor frequency scans}

Small-signal instabilities may occur due to adverse interactions between a grid and a connected PES. The main use of frequency scanning is the study of such adverse interactions. For this, the complete system is represented as the combination of two independent systems as shown in figure 26, while the closed-loop frequency response of the system may be obtained as shown in figure 26 . The closedloop transfer function is $Z_{e q}(j \omega)=\left(Z_{G R I D}(j \omega)^{-1}+\right.$ $\left.Y_{P E S}(j \omega)\right)^{-1} \cdot Z_{G R I D}(j \omega)$ and $Y_{P E S}(j \omega)$ are the impedance and admittance matrices of the grid and PES respectively ${ }^{1}$, the latter being obtained using frequency scanning, while the former may be obtained using an analytical model or by frequency scanning. Closed-loop Nyquist Criterion may be used to check the stability of this closed-loop system provided both the model of Grid and PES are LTI.

In both DQ and Dynamic Phasor based analyses, $Z_{G R I D}$ and $Y_{P E S}$ are matrix transfer functions. Therefore, the generalized Nyquist Criterion (GNC) is used to check the stability of the closed-loop feedback system.

\footnotetext{
${ }^{1}$ For devices like TCSC, the dual form is used with $Z_{P E S}$ and $Y_{G R I D}$.
} 

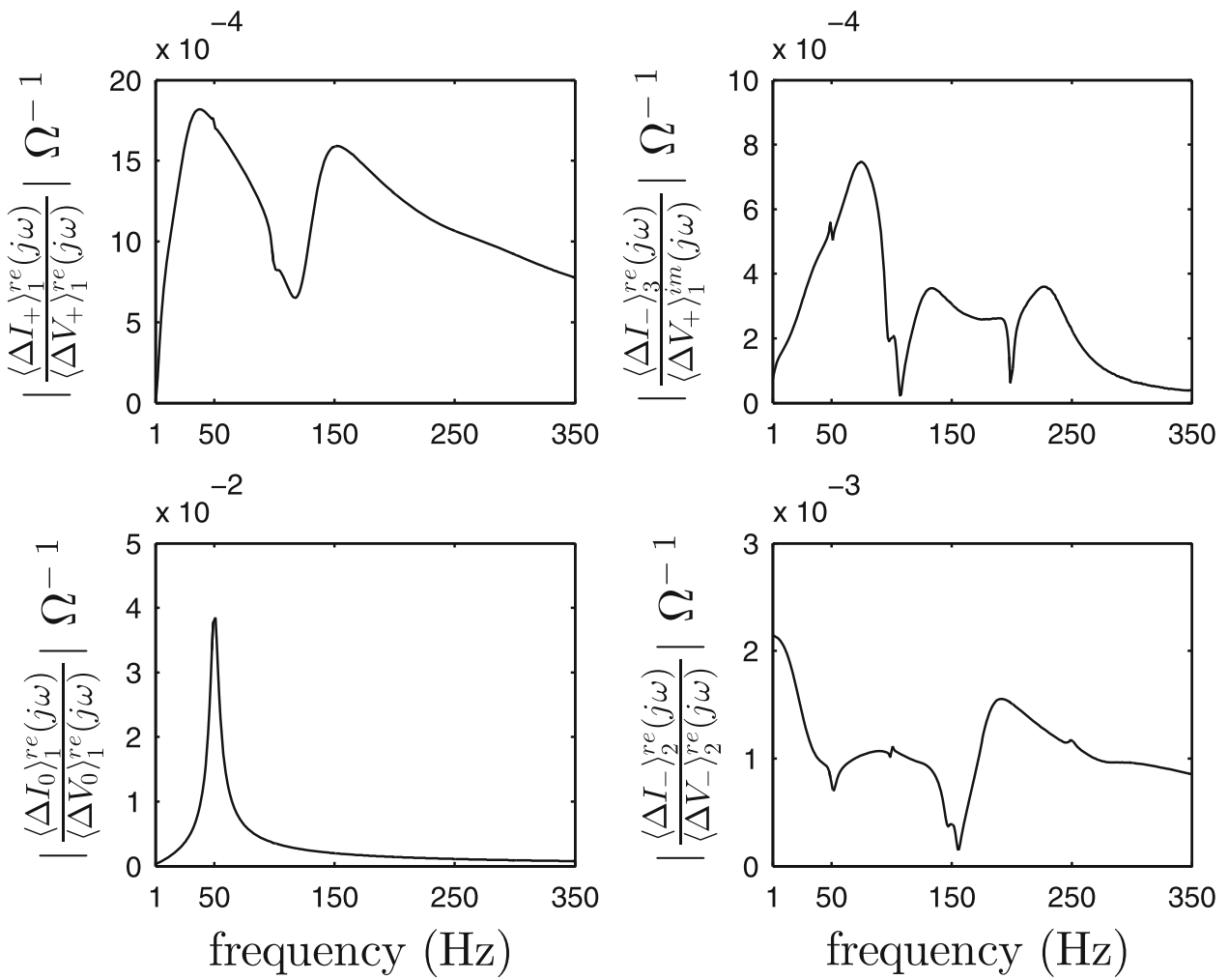

Figure 25. Frequency response obtained from a dynamic phasor based scan of a PWM-switched STATCOM with a sequence based controller.

Table 1. Inter-convertibility among phase/sequence, DQ and dynamic phasor model for different type of systems (NA: not-applicable).

\begin{tabular}{|c|c|c|c|c|c|}
\hline System & Example & $\begin{array}{l}\text { Phase/ } \\
\text { sequence } \\
\text { model }\end{array}$ & $\begin{array}{c}\text { DQ } \\
\text { model }\end{array}$ & $\begin{array}{l}\text { Dynamic phasor } \\
\text { model in phase/ } \\
\text { sequence variables }\end{array}$ & Remark \\
\hline \multicolumn{6}{|l|}{ No switching elements } \\
\hline Three-phase balanced system & $\begin{array}{c}\text { Balanced inductor and } \\
\text { capacitor banks, } \\
\text { transmission lines }\end{array}$ & $\begin{array}{l}\text { LTI } \\
\text { (diagonal) }\end{array}$ & LTI & $\begin{array}{r}\text { LTI (block } \\
\text { diagonal) }\end{array}$ & $\begin{array}{l}\text { Models are completely inter- } \\
\text { convertible }\end{array}$ \\
\hline Three-phase unbalanced system & $\begin{array}{l}\text { Unbalanced inductor } \\
\text { and capacitor } \\
\text { banks,transmission } \\
\text { line }\end{array}$ & $\begin{array}{l}\text { LTI } \\
\text { (sequence } \\
\text { coupling) }\end{array}$ & $\begin{array}{l}\text { Not } \\
\text { LTI }\end{array}$ & $\begin{array}{r}\text { LTI (block } \\
\text { diagonal) }\end{array}$ & $\begin{array}{l}\text { Phase model and dynamic } \\
\text { phasor model are inter- } \\
\text { convertible }\end{array}$ \\
\hline Single-phase & $\begin{array}{l}\text { Single pahse inductor, } \\
\text { capacitor, } \\
\text { transmission line }\end{array}$ & LTI & NA & $\begin{array}{r}\text { LTI (block } \\
\text { diagonal) }\end{array}$ & $\begin{array}{l}\text { Phase model and dynamic } \\
\text { phasor model are inter- } \\
\text { convertible }\end{array}$ \\
\hline \multicolumn{6}{|l|}{ With switching elements } \\
\hline Single-phase converter & $\begin{array}{c}\text { Single phase rectifier, } \\
\text { inverter }\end{array}$ & Not LTI & NA & LTI & \\
\hline $\begin{array}{l}\text { Balanced three- phase converter } \\
\text { with negligible harmonics in } \\
\text { switching function }\end{array}$ & $\begin{array}{c}\text { PWM switched } \\
\text { STATCOM }\end{array}$ & Not LTI & LTI & LTI & $\begin{array}{l}\text { DQ to phase model (at } \\
\text { complementary } \\
\text { frequencies) is possible }\end{array}$ \\
\hline $\begin{array}{l}\text { Balanced three-phase converter } \\
\text { with significant harmonics in } \\
\text { switching function }\end{array}$ & $\begin{array}{c}\text { 12-pulse STATCOM, } \\
\text { TCSC, HVDC }\end{array}$ & Not LTI & $\begin{array}{l}\text { Not } \\
\text { LTI }\end{array}$ & LTI & $\begin{array}{l}\text { Number of harmonic } \\
\text { dynamic phasors to be } \\
\text { considered requires further }\end{array}$ \\
\hline $\begin{array}{l}\text { Unbalanced three-phase } \\
\text { converter }\end{array}$ & $\begin{array}{l}\text { STATCOM with } \\
\text { sequence based } \\
\text { controller }\end{array}$ & Not LTI & $\begin{array}{l}\text { Not } \\
\text { LTI }\end{array}$ & LTI & analysis \\
\hline
\end{tabular}




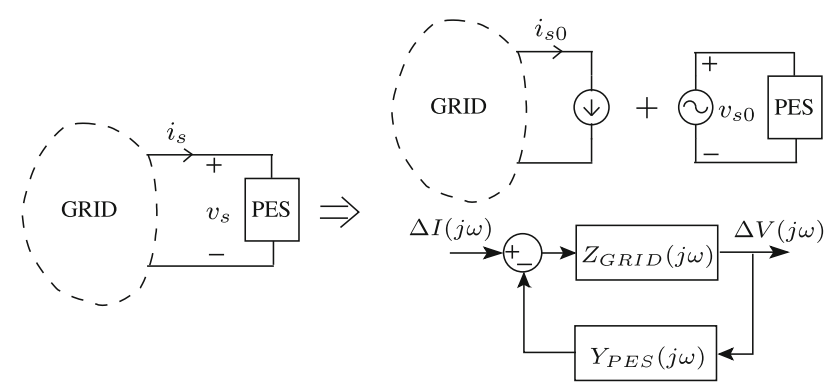

Figure 26. PES connected to grid.

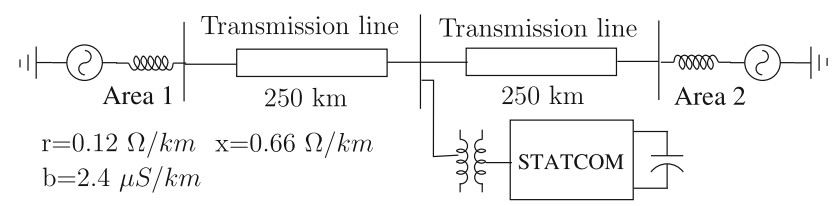

Figure 27. STATCOM-connected network.

\subsection{Generalized Nyquist criterion [26]}

If $Z_{G R I D}(j \omega)$ and $Y_{P E S}(j \omega)$ are stable systems, the closedloop system is stable provided there is no encirclement of the contour of $\Delta(j \omega)=\operatorname{det}\left(I+Z_{G R I D}(j \omega) Y_{P E S}(j \omega)\right)$ around the origin of complex plane as $\omega$ traverses from $-\infty$ to $\infty$.

The transfer functions obtained from a Dynamic Phasor model are equivalent to 'harmonic transfer functions' of time-periodic systems. According to the property of harmonic transfer functions [27], the contour of $\Delta(j \omega)$ repeats itself at every $\omega_{0}$, where $\omega_{0}$ is the fundamental frequency. Therefore, in dynamic phasor based analysis, it is adequate to evaluate the contour of $\Delta(j \omega)$ from $-\omega_{0} / 2$ to $+\omega_{0} / 2$ to obtain the required encirclement information.

\subsection{Case studies}

In this section, we check the ability of the frequency scan models of PES obtained using dynamic phasor variables for accurate prediction of stability using the GNC.

6.2a STATCOM-network interaction: Figure 27 shows a $400 \mathrm{kV}, 50 \mathrm{~Hz}, \pm 200 \mathrm{MVA}$ STATCOM connected at the mid-point of a $500 \mathrm{~km}$ transmission system. The STATCOM is equipped with the Delay Signal Cancellation (DSC) based positive and negative sequence controllers [22], and uses sinusoidal PWM technique with a carrier frequency of $1050 \mathrm{~Hz}$. The STATCOM operates in the constant reactive current control mode. Controller data are given in Appendix I.

The frequency responses of the STATCOM and the network are independently obtained by frequency scanning, and GNC is used to check the stability of the combined system. Both stable and unstable operating points are obtained by changing

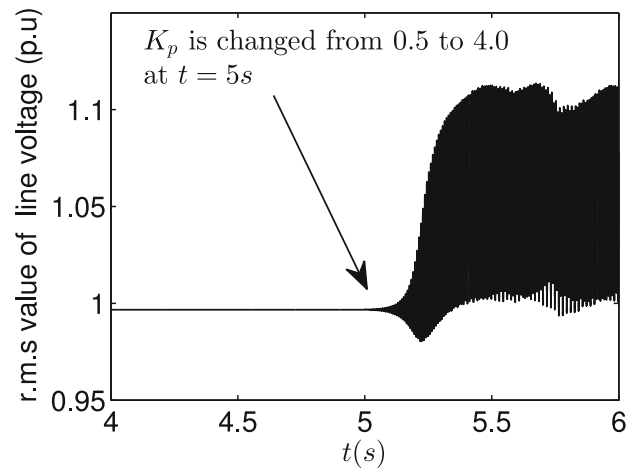

Figure 28. Response of r.m.s value of STATCOM line voltage.

the proportional gain of DC voltage controller from $K_{p}=0.5$ to $K_{p}=4.0 \mathrm{pu} / \mathrm{pu}$. This can be seen from the response of the STATCOM terminal voltage shown in figure 28.

For dynamic phasor based frequency scanning, a multisine input of magnitude $0.25 \mathrm{kV}$, with a frequency resolution of $0.1 \mathrm{~Hz}$ is used. The frequency response in dynamic phasor variables is obtained from $0.1 \mathrm{~Hz}$ to $25 \mathrm{~Hz}$ (see the discussion on GNC in the previous section) and harmonics up to $k=8$ are considered. Consistent results from GNC are obtained for $k>7$ (see figures 29 and 30). The Nyquist contour obtained for the stable case is shown in figure 30(a). Net encirclement is zero. According to GNC, the system is stable, which is consistent with the time-domain simulation of the combined system.

Similarly for the unstable case, the Nyquist contour is shown in figure 30(b). There are two clock-wise encirclements $(N=2)$. Therefore, the closed-loop system is unstable according to GNC, which is consistent with the results of the time-domain simulation of the combined system.

6.2b Network mode instability in a TCSC compensated system: Figure 31 shows the single line diagram of a series compensated transmission line. The TCSC control is of individual-phase type, with a reactance controller and an auxiliary SSR damping controller (SSDC) [28]. For the control of the TCSC reactance, an individual phase control (IPC) scheme is used; the phase $a$ controller is shown in figure 32(a). A quarter cycle delay based method is used to extract the in-phase and quadrature vector components of current and voltage of an individual phase, as shown in figure 32(b) and 32(c). The Phase Locked Loop (PLL) for phase $a$ shown in figure 32(d) locks on to the phase $a$ line current by driving its quadrature component to zero.

The TCSC controller is equipped with a SSR damping controller (SSDC) which is used to modulate the firing angle order generated by the reactance controller. The structure of a typical SSDC is shown in figure 33. It consists of a gain block, a low pass filter, a washout block and a compensator. The input to the SSDC is line current magnitude. ${ }^{2}$

\footnotetext{
${ }^{2}$ Instantaneous current magnitude is computed as follows: $I=\sqrt{i_{a}^{2}+i_{b}^{2}+i_{c}^{2}}$.
} 


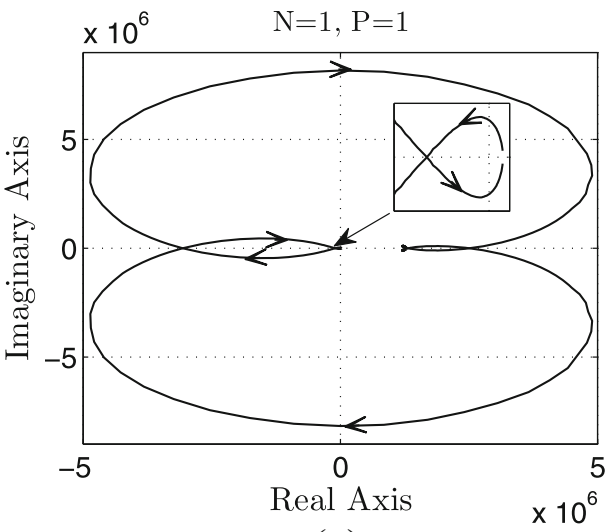

(a)

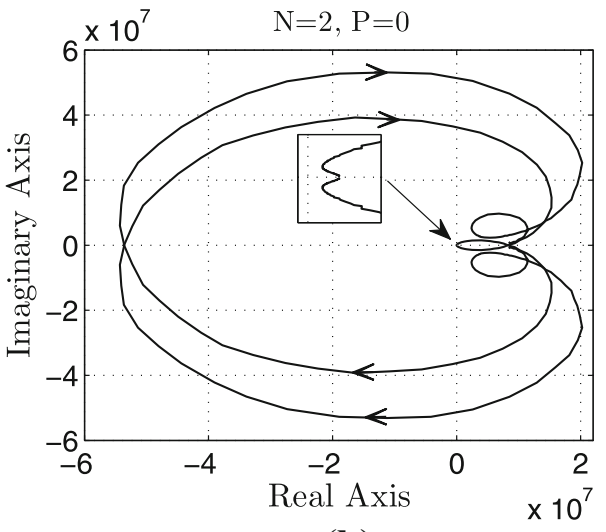

(b)

Figure 29. Nyquist contour with dynamic phasor based model $(k=7)$ of STATCOM: (a) stable and (b) unstable.

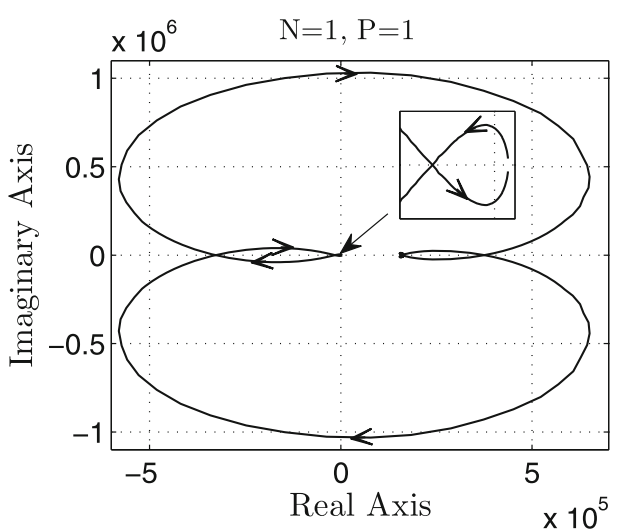

(a)

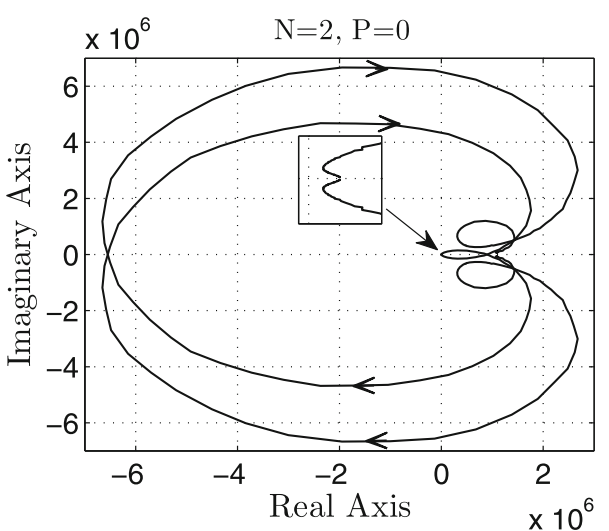

(b)

Figure 30. Nyquist contour with dynamic phasor based model $(k=8)$ of STATCOM: (a) stable and (b) unstable.

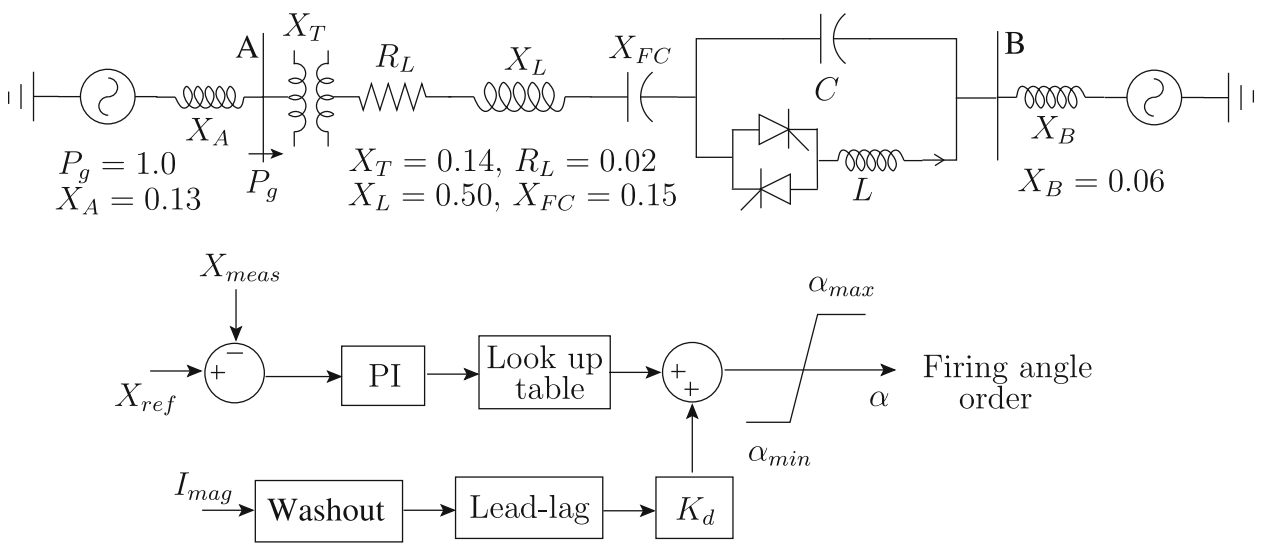

Damping controller

Figure 31. TCSC-compensated electrical network. 


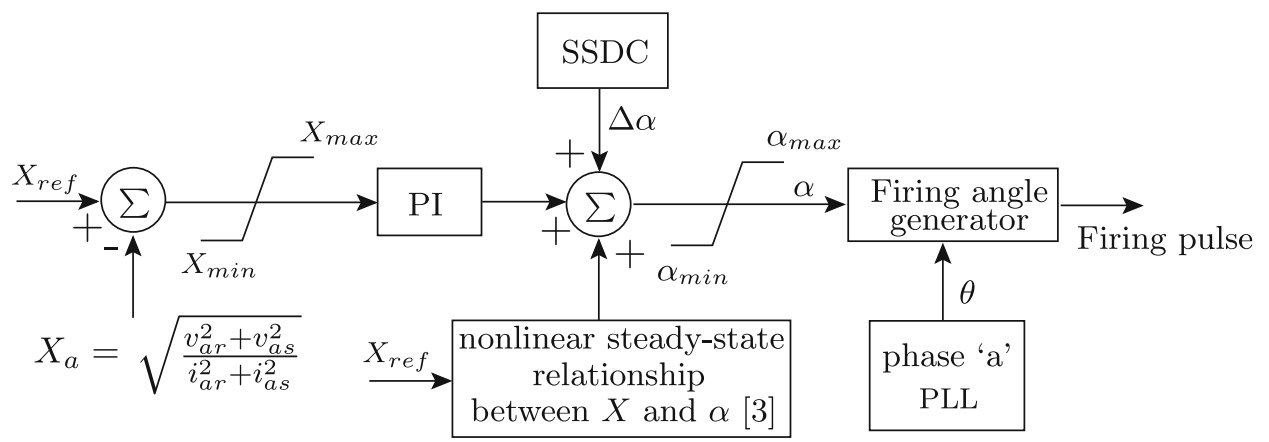

(a)

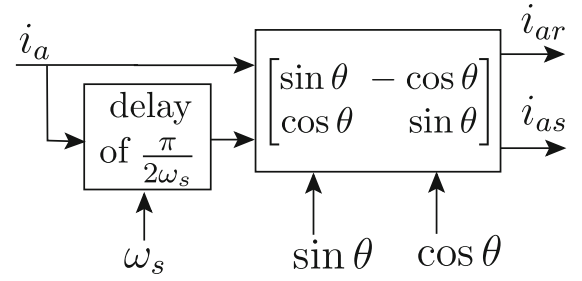

(b)

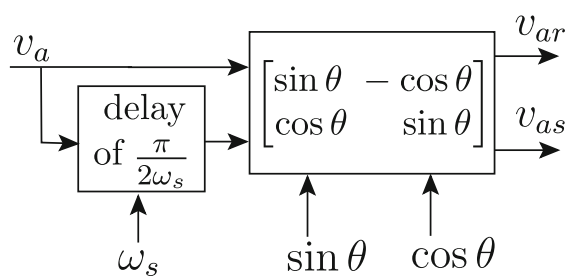

(c)

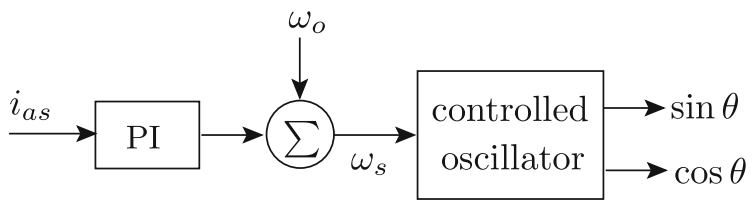

(d)

Figure 32. (a) TCSC controller, (b) extraction of vector components for line current, (c) extraction of vector components for TCSC capacitor voltage and (d) PLL for phase $a$.

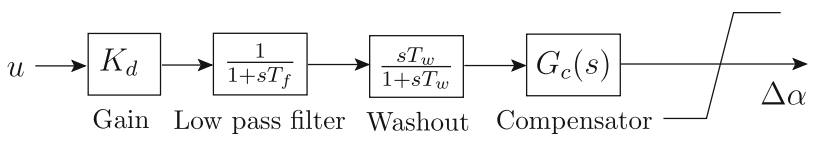

Figure 33. SSDC structure.
The magnitude of multi-sine used for frequency scanning of the TCSC is $80 \mathrm{~mA}$ with a gap of $0.1 \mathrm{~Hz}$. The dynamic phasor scan is obtained from $0.1 \mathrm{~Hz}$ to $30 \mathrm{~Hz}$. Harmonics up to $k=6$ is considered.

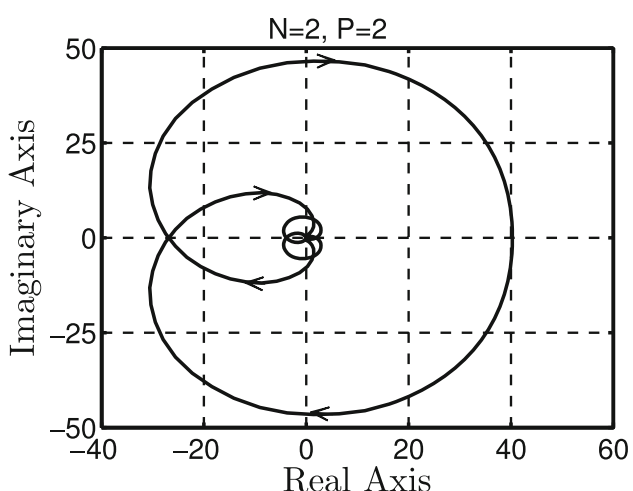

(a)

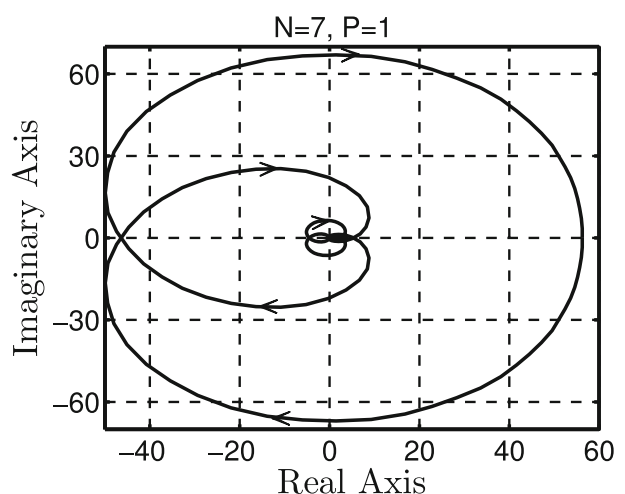

(b)

Figure 34. Nyquist contour with dynamic phasor based model of TCSC: (a) Stable and (b) unstable. 


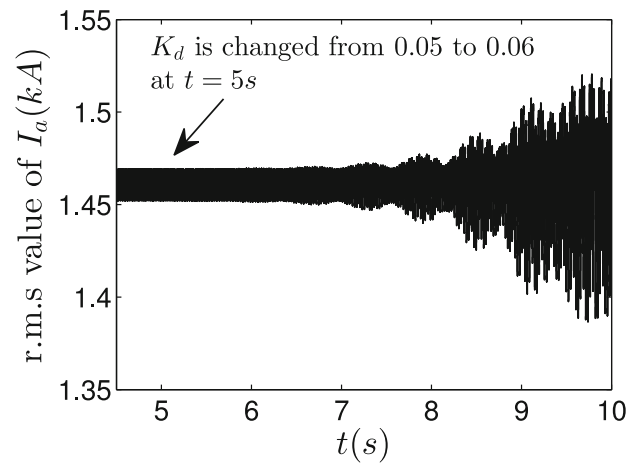

Figure 35. Response of rms value of phase-a of the line current.

Stable and unstable cases are obtained by changing the gain of the controller from $K_{d}=0.05$ to $K_{d}=0.06 \mathrm{rad} / \mathrm{pu}$ as shown in figure 35 .

For the stable case, the Nyquist contour based on Dynamic phasor model is shown in figure 34(a). Since there is no encirclement, the closed loop system is stable. For the unstable case, the Nyquist contour plot shown in figure 34(b), has encirclements around the origin. This indicates closed loop system is unstable. Thus, the model accurately predicts the stability behavior of the system.

\section{Discussion}

Dynamic phasor based frequency scanning is the most general technique for PES, with no constraints or assumptions on the nature of the controllers or switching frequency, as long as the systems are time-periodic. The scans are smooth due to the time-invariance of the underlying model in the dynamic phasor variables, which facilitates its use for further analysis. This technique has a few disadvantages, which are discussed here.

Dynamic phasor based scanning requires a larger number of time domain simulations as compared to phase/sequence or DQ based scanning. The number of simulations increases if the frequency scanning range is increased or if the transfer functions corresponding to a larger number of dynamic phasor components $(k)$ are included.

The use of the frequency responses of the dynamic phasor variables for small-signal stability studies is unambiguous because the underlying system is time-invariant. The responses are generally smooth, but are in the form of a matrix transfer function (Multi-Input, Multi-Output MIMO), whose dimension is determined by the number of harmonic components $(k)$ which are considered.

\section{Conclusion}

In this paper we propose and present the methodology of dynamic phasor based frequency scanning. Since most PES are time-periodic, they result in a time-invariant model when expressed in terms of dynamic phasor variables. Unlike phase/sequence or DQ variables based scanning, the frequency scans in terms of dynamic phasors are smooth even for systems with low-order switching harmonics (like 12-pulse systems, TCSC) or with negative sequence controllers, due to the time-invariance property. These aspects are brought out in the paper through illustrative examples of TCSC and STATCOM. In the case of a TCSC, we also compare the frequency responses obtained by scanning with those obtained using an approximate analytical dynamic phasor model. The frequency responses match at lower frequencies, where the approximations are valid.

Further use of the scans of time-periodic power electronic systems for stability analysis is facilitated by the time-invariance property of the underlying model in dynamic phasor variables. For dynamic interaction studies, the frequency responses of a PES obtained using dynamicphasor scanning can be combined with analytically or numerically derived frequency responses (in dynamic phasor variables) of the network to which it is connected. Stability of the combined system is evaluated using the Generalized Nyquist criterion. Grid interaction studies using GNC give accurate result if a sufficient number of dynamic phasors are considered. Additional investigations are required to obtain the guidelines for the approximate choice of the number of dynamic phasors $(k)$ in a general situation.

The use of frequency scan models for eigen-value analysis and parametric sensitivity analysis also is a subject for future research.

\section{Acknowledgements}

The authors wish to thank the Department of Electronics and Information Technology, Government of India, for financial support to carry out this work, under the project 'Simulation Centre for Power Electronics and Power Systems'.

\section{Appendix I. STATCOM with a negative sequence controller}

See Tables 2 and 3.

Table 2. Positive sequence real and reactive power controller parameters.

\begin{tabular}{lccc}
\hline Parameters & $v_{d c}$ controller & $i_{q+}$ controller & $i_{d+}$ controller \\
\hline$K_{p}$ & $0.5 \mathrm{pu} / \mathrm{pu}$ & $0.19 \mathrm{pu} / \mathrm{pu}$ & $0.19 \mathrm{pu} / \mathrm{pu}$ \\
$K_{i}$ & $0.2 \mathrm{pu} / \mathrm{pu}-\mathrm{s}$ & $5.46 \mathrm{pu} / \mathrm{pu}-\mathrm{s}$ & $5.46 \mathrm{pu} / \mathrm{pu}-\mathrm{s}$ \\
\hline
\end{tabular}


Table 3. Negative sequence current controller parameters.

\begin{tabular}{lcc}
\hline Parameters & $i_{q-}$ controller & $i_{d-}$ controller \\
\hline$K_{p}$ & $0.19 \mathrm{pu} / \mathrm{pu}$ & $0.19 \mathrm{pu} / \mathrm{pu}$ \\
$K_{i}$ & $5.16 \mathrm{pu} / \mathrm{pu}-\mathrm{s}$ & $5.46 \mathrm{pu} / \mathrm{pu}-\mathrm{s}$ \\
\hline
\end{tabular}

\section{References}

[1] Ainsworth J D 1967 Harmonic instability between controlled static converters and ac networks. Proc. IEE 114(7): 949-957

[2] Padiyar K R 1999 Analysis of subsynchronous resonance in power systems. Norwell, MA: Kluwer

[3] Irwin G D, Jindal A K and Isaacs A L 2011 Sub-synchronous control interactions between type 3 wind turbines and series compensated ac transmission systems. In: Proc. IEEE PES General Meeting, San Diego, CA, pp. 1-6

[4] Schauder C and Mehta H 1993 Vector analysis and control of advanced static VAR compensators. IEE Proc. C 140(4): 299-306

[5] Mattavelli P Verghese G C and Stankovic A M 1997 Phasor dynamics of thyristor-controlled series capacitor systems. IEEE Trans. Power Syst. 12(3): 1259-1269

[6] Jalali S G, Lasseter R H and Dobson I 1994 Dynamic response of a thyristor controlled switched capacitor. IEEE Trans. Power Delivery 9(3): 1609-1615

[7] Jiang X and Gole A M 1995 A frequency scanning method for identification of harmonic instabilities in HVDC systems. IEEE Trans. Power Delivery 10(4): 1875-1881

[8] Das M K, Kulkarni A M and Gole A M 2012 A screening technique for anticipating network instabilities in AC-DC systems using sequence impedances obtained by frequency scanning. In: 10th IET International Conference on AC and DC Power Transmission (ACDC 2012), Birmingham, UK, pp. 1-6

[9] Wang X, Blaabjerg F and Wu W 2014 Modeling and analysis of harmonic stability in an ac power-electronics-based power system. IEEE Trans. Power Electron. 29(12): 6421-6432

[10] Sanchez S, Bergna G, Berne E, Egrot P, Vannier J -C and Molinas M 2013 Frequency scanning of power electronicbased smart grids: the modular multilevel converter application. In: 4th IEEE International Symposium on Power Electronics for Distributed Generation Systems (PEDG), Rogers, AR, USA, pp. 1-8

[11] Sun J 2011 Impedance-based stability criterion for gridconnected inverters. IEEE Trans. Power Electron. 26(11): 3075-3078
[12] Fazeli S M, Ping H W, Rahim N B and Ooi B T 2013 Individual-phase decoupled PQ control of three phase voltage source converter. IET Gener. Transm. Distrib. 7(11): 1219-1228

[13] Hochgraf C and Lasseter R H 1998 STATCOM controls for operation with unbalanced voltages. IEEE Trans. Power Delivery 13(2): 538-544

[14] Chudasama M C 2012 Application of dynamic phasor models for the analysis of power systems with phase imbalance. Ph.D. dissertation, Dept. of Elect. Eng., Indian Institute of Technology, Bombay, India

[15] Pintelon R and Schoukens J 2001 System identification: a frequency domain approach. Piscataway, NJ: IEEE Press

[16] Oppenheim A V and Schafer R W 1989 Discrete-time signal processing. NJ: Prentice-Hall

[17] PSCAD/EMTDC Users guide 2010 Manitoba HVDC Research Centre. Winnipeg, Canada

[18] Padiyar K R and Kulkarni A M 1997 Design of reactive current and voltage controller of static condenser. Int. J. Electr. Power Energy Syst. 19(6): 397-410

[19] Mohaddes M, Gole A M and Elez S 2001 Steady state frequency response of STATCOM. IEEE Trans. Power Deliv. 16(1): 18-23

[20] Mathworks. Inc., MATLAB Users guide, 2010

[21] Yazdani A and Iravani R 2006 A unified dynamic model and control for the voltage-sourced converter under unbalanced grid conditions. IEEE Trans. Power Deliv. 21(3): 1620-1629

[22] Jiang Y and Ekstrom A 1997 Applying PWM to control over currents at unbalanced faults of force- commutated VSCs used as static var compensators. IEEE Trans. Power Deliv. 12(1): 273-278

[23] Salunkhe K and Kulkarni A M 2015 Reconstruction of transient waveforms from phasors sampled at the fundamental frequency. Eindhoven: IEEE PowerTech, pp. 1-6

[24] Del Rosso A D, Caizares C A and Doa V M 2003 A study of TCSC controller design for power system stability improvement. IEEE Trans. Power Syst. 18(4): 1487-1496

[25] Dermiray T H 2008 Simulation of power system dynamics using dynamic phasor models. Ph.D. dissertation, Dept. Inf. Technol. Elect. Eng., Swiss Federal Institute of Technology, Zurich, Switzerland

[26] MacFarlane A G J and Postlethwaite I 1997 The generalized Nyquist stability criterion and multivariable root loci. Int. J. Control 25(1): 81-127

[27] Wereley N 1991 Analysis and control of linear periodically time varying systems. Ph.D. dissertation, Dept. Aero. Astro., Mass. Inst. Technol., Cambridge, MA

[28] Joshi S R and Kulkarni A M 2009 Analysis of SSR performance of TCSC control schemes using a modular high bandwidth discrete-time dynamic model. IEEE Trans. Power Syst. 24(2): 840-848 